\title{
Adaptation of Saccharomyces cerevisiae to saline stress through laboratory evolution
}

\author{
Dhar, R ; Sägesser, R ; Weikert, C ; Yuan, J ; Wagner, A
}

\begin{abstract}
Most laboratory evolution studies that characterize evolutionary adaptation genomically focus on genetically simple traits that can be altered by one or few mutations. Such traits are important, but they are few compared with complex, polygenic traits influenced by many genes. We know much less about complex traits, and about the changes that occur in the genome and in gene expression during their evolutionary adaptation. Salt stress tolerance is such a trait. It is especially attractive for evolutionary studies, because the physiological response to salt stress is well-characterized on the molecular and transcriptome level. This provides a unique opportunity to compare evolutionary adaptation and physiological adaptation to salt stress. The yeast Saccharomyces cerevisiae is a good model system to study salt stress tolerance, because it contains several highly conserved pathways that mediate the salt stress response. We evolved three replicate lines of yeast under continuous salt $(\mathrm{NaCl})$ stress for 300 generations. All three lines evolved faster growth rate in high salt conditions than their ancestor. In these lines, we studied gene expression changes through microarray analysis and genetic changes through next generation population sequencing. We found two principal kinds of gene expression changes, changes in basal expression (82 genes) and changes in regulation (62 genes). The genes that change their expression involve several well-known physiological stress-response genes, including CTT1, MSN4 and HLR1. Next generation sequencing revealed only one high-frequency single-nucleotide change, in the gene MOT2, that caused increased fitness when introduced into the ancestral strain. Analysis of DNA content per cell revealed ploidy increases in all the three lines. Our observations suggest that evolutionary adaptation of yeast to salt stress is associated with genome size increase and modest expression changes in several genes.
\end{abstract}

DOI: https://doi.org/10.1111/j.1420-9101.2011.02249.x

Posted at the Zurich Open Repository and Archive, University of Zurich

ZORA URL: https://doi.org/10.5167/uzh-59820

Journal Article

Accepted Version

Originally published at:

Dhar, R; Sägesser, R; Weikert, C; Yuan, J; Wagner, A (2011). Adaptation of Saccharomyces cerevisiae to saline stress through laboratory evolution. Journal of Evolutionary Biology, 24(5):1135-1153.

DOI: https://doi.org/10.1111/j.1420-9101.2011.02249.x 


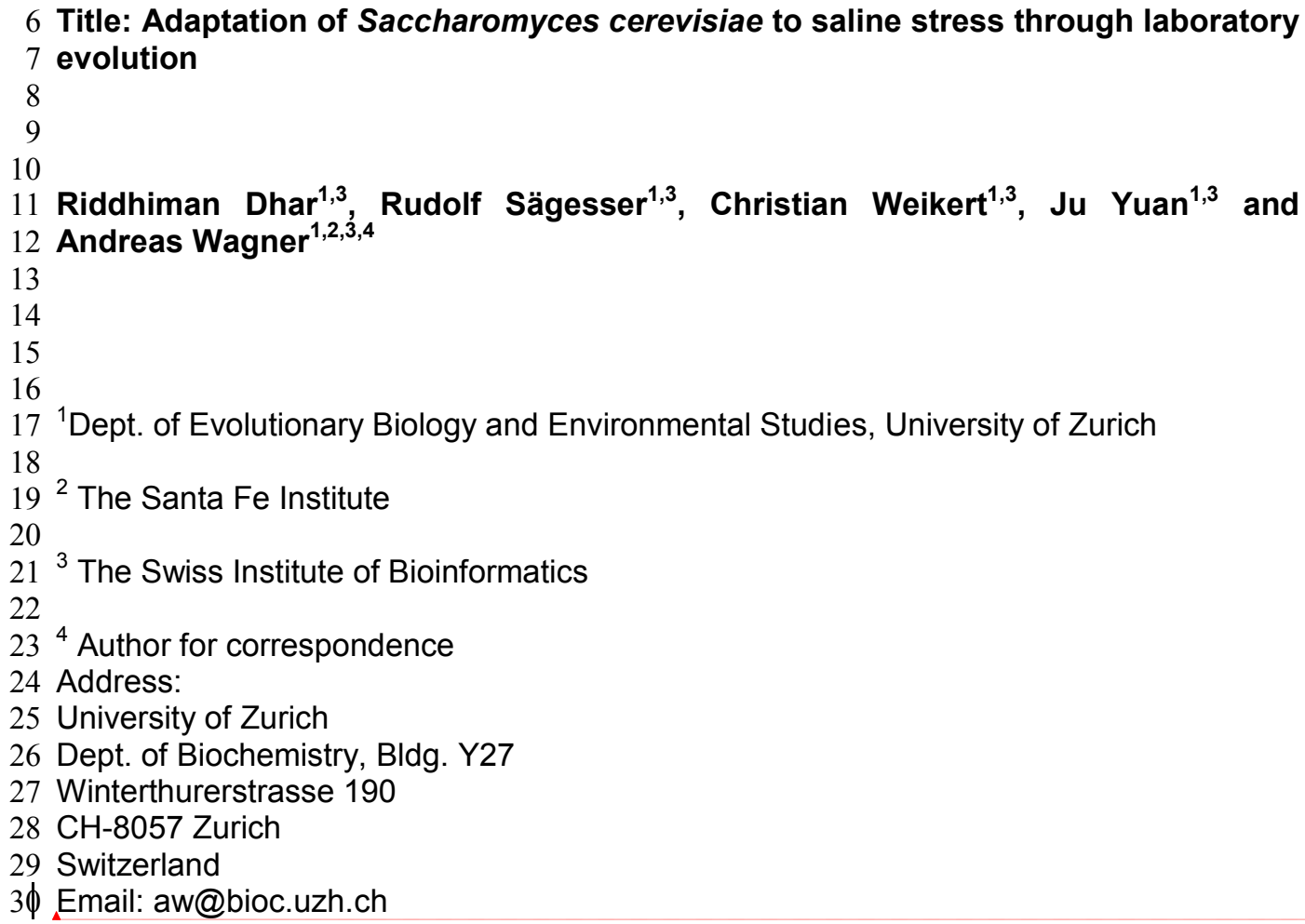

6 Title: Adaptation of Saccharomyces cerevisiae to saline stress through laboratory 7 evolution

11 Riddhiman Dhar ${ }^{1,3}$, Rudolf Sägesser
12 Andreas Wagner
$1,2,3,4$

$17{ }^{1}$ Dept. of Evolutionary Biology and Environmental Studies, University of Zurich

$19^{2}$ The Santa Fe Institute

$21^{3}$ The Swiss Institute of Bioinformatics

$23{ }^{4}$ Author for correspondence

25 University of Zurich

26 Dept. of Biochemistry, Bldg. Y27

27 Winterthurerstrasse 190

$28 \mathrm{CH}-8057$ Zurich

29 Switzerland

$3 \phi$ Email: aw@bioc.uzh.ch 13

18

20

22

24 Address:

31 Phone: +41-44-635-6141

32 Fax: +41-44-635-6144

33

34

\section{Abstract}

36 Most laboratory evolution studies that characterize evolutionary adaptation genomically

37 focus on genetically simple traits that can be altered by one or few mutations. Such

38 traits are important, but they are few compared to complex, polygenic traits influenced

39 by many genes. We know much less about complex traits, and about the changes that 40 occur in the genome and in gene expression during their evolutionary adaptation. Salt

41 stress tolerance is such a trait. It is especially attractive for evolutionary studies,

42 because the physiological response to salt stress is well-characterized on the molecular 43 and transcriptome level. This provides a unique opportunity to compare evolutionary 44 adaptation and physiological adaptation to salt stress. The yeast Saccharomyces 
45 cerevisiae is a good model system to study salt stress tolerance, because it contains 46 several highly conserved pathways that mediate the salt stress response. We evolved 47 three replicate lines of yeast under continuous salt $(\mathrm{NaCl})$ stress for 300 generations. All 48 three lines evolved faster growth rate in high salt conditions than their ancestor. In these 49 lines, we studied gene expression changes through microarray analysis, and genetic 50 changes through next generation population sequencing. We found two principal kinds 51 of gene expression changes, changes in basal expression (82 genes), and changes in 52 regulation (62 genes). The genes that change their expression involve several well53 known physiological stress response genes, including CTT1, MSN4, HLR1. Next 54 generation sequencing revealed only one high frequency single nucleotide change, in 55 the gene MOT2, that caused increased fitness when introduced into the ancestral strain. 56 Analysis of DNA content per cell revealed ploidy increases in all the three lines. Our 57 observations suggest that evolutionary adaptation of yeast to salt stress is associated 58 with genome size increase and modest expression changes in several genes.

\section{Introduction}

70 The ability to characterize the changes that occur during evolutionary adaptation on a

71 genome-wide scale has been a boon for the field of laboratory evolution. Most published

72 studies focus on traits with a simple basis, where changes of major effects in one or few

73 changes can alter a trait during laboratory evolution experiments (Ferea et al., 1999;

74 Blanc \& Adams, 2003; Velicer et al., 2006; Stanek et al., 2009). Such traits are

75 important, but they are in the minority. The vast majority of traits have a complex,

76 polygenic basis (refs. Benfey, Genomics textbook). We know much less about how

77 genomic change and change in gene expression occurs in such polygenic traits. Our

78 study is a step towards answering this question. We here focus on a prototypical

79 polygenic trait, an organism's response to high concentrations of salt in its environment.

80 The physiological response of an organism to such salt stress is well-studied on the

81 molecular and the transcriptome levels (Posas et al., 2000; Rep et al., 2000; Causton et 82 al., 2001; Yale \& Bohnert, 2001). This fact provides another important motivation to

83 study the salt stress response in an evolutionary context. It allows us to ask whether 84 evolutionary adaptation to salt stress is similar to physiological adaptation, using a 85 genome-scale approach that relies on transcriptome changes in response to salt stress. 86

87 the wild. Organisms thus use cellular mechanisms to adapt to and to survive 88 environmental fluctuations. Hyperosmotic stress is one prominent environmental 
89 stressor, where a cell experiences higher solute concentration outside the cell than

90 inside. This causes water loss from the cell, resulting in a higher intracellular

91 concentration of ions and metabolites, and eventual arrest of cellular activity.

92 Hyperosmotic stress is caused by high concentrations of sugar or salt, e.g., sodium

93 chloride $(\mathrm{NaCl})$. High salt stress is a special case of hyperosmotic stress and has similar

94 effects on a cell as high concentration of sugars (Gasch et al., 2000; Causton et al.,

95 2001). In addition, it causes hyperionic stress due to high extracellular concentrations of

$96 \mathrm{Na}^{+}$and $\mathrm{Cl}^{-}$ions, which are imported into the cell and can disrupt cellular ionic

97 equilibrium. Tolerance to $\mathrm{Na}^{+}$stress thus needs additional ion transport and

98 detoxification mechanisms along with those required for the hyperosmotic stress

99 response (Serrano, 1996; Apse et al., 1999; Gaxiola et al., 1999; Maathuis \& Amtmann,

100 1999; Serrano et al., 1999; Zhu, 2001). The hyperosmotic stress response in yeast is

101 mediated by the high osmolarity glycerol (HOG) pathway, which is a MAPK pathway

102 (Brewster et al.,1993; Dihazi et al., 2004, Saito \& Tatebayashi, 2004; reviewed in

103 Hohmann 2002).Two cell membrane-bound sensors Sho1p and SIn1p detect osmotic

104 change, which results in activation of Hog pathway genes, which, in turn, leads to the

105 activation of the downstream genes associated with salt tolerance and adaptation

106 (Martinez-Pastor et al., 1996; Schmitt \& McEntee, 1996; Gorner et al., 1998; Ostrander

107 \& Gorman, 1999; Rep et al., 1999; Reiser et al., 2000; Rep et al., 2000). Whole

108 transcriptome studies by Gasch et al, Posas et al, Yale and Bonhart, Causton et al., and

109 Rep et al. (Gasch et al., 2000; Posas et al., 2000; Rep et al., 2000; Causton et al., 2001;

110 Yale \& Bohnert, 2001) have identified hundreds of genes whose expression levels are

111 affected by hyperosmotic stress. The genes induced during osmotic stress response

112 include the genes involved in synthesis and regulation of the cellular osmolytes glycerol

113 and trehalose. Another group of genes, specifically activated under saline stress, are

114 associated with ion homeostasis. Some of the genes affected by osmotic stress show

115 transient expression changes. whereas others show expression changes that are stable

116 in time. In addition to genome wide approaches, many studies have characterized the

117 roles of individual genes in the osmotic stress response of yeast (Haro et al., 1991;

118 Garciadeblas et al., 1993; Mai \& Breeden, 1997; Ganster et al., 1998; Mendizabal et

119 al.,1998; Tsujimoto et al., 2000; Betz et al., 2002; Goossens et al., 2002; Hirata et al.,

120 2003; Heath et al., 2004; ).

121 Yeast is a good model system for studying osmo-adaptation in eukaryotes,

122 since other fungi and plants share many of the stress response pathways and proteins

123 involved in osmo-adaptation in yeast. First, the mitogen-activated protein kinase (MAP

124 kinase) cascade, central to the stress response in yeast, is a conserved eukaryotic

125 signal transduction pathway present from fungi to plants (Pelech \& Sanghera, 1992;

126 Errede \& Levin, 1993; Nishida \& Gotoh, 1993; Neiman, 1993; Neiman et al., 1993;

127 Kosako et al., 1993; Tsuda et al., 1993; Yashar et al., 1993; Cooper, 1994; Zamanillo et

128 al., 1994; Sanchez et al., 1994; Yan et al., 1994; Galcheva-Gargova et al., 1994; Han et

129 al., 1994; Waskiewicz \& Cooper, 1995; Hirt, 1997; Jonak et al.,1999; Kovtun et al.,

130 2000; Kyriakis \& Avruch, 2001). Stress signaling in plants is also carried out by MAPK

131 pathways, which are activated by cold, drought, salt, heat, and oxidative stress (Jonak

132 et al., 1996; Kovtun et al., 2000; Teige et al., 2004). Second, yeast and plants have 
133 highly similar genes required for stress tolerance (Mendoza et al., 1994; Bressan et al., 134 1998; Pardo et al., 1998; Lee et al., 1999; Sanders, 2000; Hasegawa \& Bressan, 2000;

135 Quintero et al., 2002; Zhu, 2002). Third, yeast and plants have similar membrane ion

136 transport and detoxification systems (Gaxiola et al., 1999; Quinteroa et al., 2000). For

137 example, HAL family genes are important for ion homeostasis in yeast as well as in

138 plants (Haro et al., 1991; Gaxiola at al., 1992; Ferrando et al., 1995; Murguía et al.,

139 1996; Rios et al., 1997; Mulet et al., 1999; Espinosa-Ruiz et al., 1999; Gisbert et al.,

140 2000; Yang et al., 2001).

$141 \quad$ With one exception (Samani \& Bell, 2010), all previous studies of salt stress

142 adaptation in yeast focused on physiological adaptation. Such adaptation occurs on

143 time scales up to a few hours. The mechanisms of longer term evolutionary adaptation

144 to salt stress are not known. Such adaptations occur on time scales of hundred

145 generations or more. One aim of this study is to compare a population's evolutionary

146 response to salt stress with its physiological response on the transcriptome level. Does

147 evolutionary adaptation mirror physiological adaptation? Does it affect largely the same

148 genes as the physiological response?

149 A second aim is to investigate the genetic basis of evolutionary adaptation of 150 yeast to high saline stress, as far as this is possible for a complex trait. Does the

151 adaptation come about through accumulation of identifiable beneficial point mutations?

152 Does it involve chromosomal rearrangements as observed in evolution of yeast in

153 glucose-limited or phosphate-limited media? Or does it take place through genetic and

154 epigenetic changes altering expression of genes that help cells adapt to high salt?

155 These are some of the questions we ask.

$156 \quad$ Understanding of evolutionary principles of salt tolerance could also be

157 important for biotechnological applications. Firstly, yeast cells experience high salt

158 concentration in many industrial fermentation processes, and improvement in

159 performance of yeast in such conditions would benefit the industry immensely (Attfield,

160 1997; Trainotti \& Stambuk, 2001; Zheng et al., 2011). Secondly, the principles of salt

161 tolerance in yeast might be useful for engineering fungi and crop plants for salt

162 tolerance, as both classes of organisms share many components of their stress

163 response systems.

164 In this contribution, we exposed yeast to high salt concentrations for 300

165 generations in the laboratory in medium containing sodium chloride $(\mathrm{NaCl})$. We

166 compared the fitness and viability of the evolved lines with the starting yeast strain

167 (ancestral strain), followed by characterization of gene expression changes and genetic

168 changes such as mutations, copy number variations and chromosomal alterations.

169

\section{Results}

171 To test for evolutionary adaptation of yeast cells to osmotic stress, we carried out

172 laboratory evolution experiments through 30 serial transfers in batch cultures,

173 comprising approximately 300 cell generations (see Methods). This number of

174 generations is sufficient to show evolutionary adaptations in yeast (Adams \& Oeller,

175 1986; Adams et al., 1992; Dunham et al., 2002; Gresham et al., 2008). For our

176 experiments, we used haploid populations to avoid any potential masking of adaptively 
177 significant alleles in the diploid stage (Zeyl et al., 2003). We carried out three parallel

178 replicate evolution lines in which yeast cells grew and divided in yeast peptone medium

179 supplemented with galactose as the sole carbon source and with $0.5 \mathrm{M} \mathrm{NaCl}$ (YPGN),

180 which is a high salt medium that exposes cells to high osmolarity stress. We refer to

181 these lines as S lines (S1, S2 and S3). As a control, we also carried out three replicates

182 where the growth medium did not contain $\mathrm{NaCl}$ (lines $\mathrm{C} 1, \mathrm{C} 2$ and $\mathrm{C} 3$ ).

183

\section{Evolutionary adaptation to $\mathrm{NaCl}$}

185 Before embarking on our experiments, we asked whether $\mathrm{NaCl}$ affects the fitness of our 186 ancestral strain. Only in this case would we expect that $\mathrm{NaCl}$ exerts a selection

187 pressure to which the strain can adapt. Fitness has two main components in our

188 experiments. These are viability on the one hand, and growth or cell division rate on the

189 other hand. We found that osmotic stress does not affect viability of the ancestral strain

190 significantly (Figure 1a). Not surprisingly then, viability does not increase over the

191 course of our experiment for lines evolved on $\mathrm{NaCl}$ (Figure 1b) and for lines evolved

192 without $\mathrm{NaCl}$ (Figure 1c).

193 In contrast to viability, population growth rate is affected by salt stress, as

194 shown in supplementary figure 1 . The figure indicates that the ancestral strain grows

195 significantly more slowly in medium containing $\mathrm{NaCl}$. Thus, the fitness of the ancestral

196 strain is lower in salt compared to that of the ancestral strain in medium without any salt.

197 Having established that $\mathrm{NaCl}$ does affect the fitness of the ancestral strain through its

198 growth rate, we asked if the growth rate and thus, relative fitness $w$ (see Methods) of

199 three salt evolved lines have increased after 300 generations of evolution (Figure 2a).

200 Fitness increased significantly relative to the ancestral strain, such that the final evolved

201 lines had a relative population mean fitness between $w=1.11$ and $w=1.17$, which

202 corresponds to a decrease in the average cell doubling time between $8.2 \%$ and $12.3 \%$

203 relative to the ancestral strain (see Methods).

204 Since our high salt medium is a complex medium, it is likely that part of the

205 evolutionary response we observe also reflects adaptation to medium components

206 different from $\mathrm{NaCl}$. To ask whether this is the case, we also measured the relative

207 fitness of the three $\mathrm{S}$ lines in the control medium (without $\mathrm{NaCl}$ ). Not surprisingly, the

208 fitness in this medium had also increased (Figure $2 \mathrm{~b}$ ) relative to the ancestral strain,

209 which suggests that at least part of the evolutionary adaptation we see is not specific to

$210 \mathrm{NaCl}$ as a stressor. However, two lines of evidence show that a substantial fraction of

211 the fitness increase is specific to $\mathrm{NaCl}$. The first is that the fitness increase, when

212 measured in the absence of $\mathrm{NaCl}$, appears much lower than when measured in the

213 presence of $\mathrm{NaCl}$ (Figures 2a and 2b)(t-test, $\mathrm{p}<0.0001$ for S1, S2 and S3). The second

214 line of evidence is provided by our three control lines that had evolved without the

215 addition of $\mathrm{NaCl}$ to the medium. Figure 2a shows that in every single line the fitness

216 increase, when measured in medium with $\mathrm{NaCl}$, is consistently and significantly lower

217 than for the lines that had evolved in $\mathrm{NaCl}$ (t-test, $p<0.0001$ for comparisons between

218 S1 \& C1, S2 \& C2 and S3 \& C3). Specifically, the increase in relative fitness $w$ was at

219 least 54 percent higher in the lines evolved in $\mathrm{NaCl}$ compared to the lines evolved

220 without $\mathrm{NaCl}$. In sum, a significant and substantial part of the evolutionary adaptation 
221 we observe is due to the selection pressure provided by $\mathrm{NaCl}$.

222

223 Gene Expression analysis of the evolved lines

224 To compare the expression levels of genes in the evolved lines and in the ancestral

225 strain, we performed whole gene transcriptome analysis using yeast microarrays

226 (Affymetrix). Because previous experiments had shown that many yeast genes change

227 expression in response to salt and to other stressors (Gasch et al., 2000; Posas et al.,

228 2000; Rep et al., 2000; Causton et al., 2001; Yale \& Bohnert, 2001), we expected that

229 this would also hold for our lines. Thus, we first asked which genes respond to salt in a

230 similar manner in the ancestral strain and in all evolved lines. We will refer to such

231 genes for brevity as shared genes. For all the analyses presented hereon we used all

232 the replicates (see methods).

$233 \quad$ In all, we observed 581 shared genes that were induced in salt and 580

234 shared genes that were repressed in salt with 2-fold expression change $\left(\| \log _{2}\right.$ (fold

235 change) $\mid \geq 1$, t-test, $p$-value $<0.05$, False Discovery Rate $<10 \%)$. Figure 3a shows a

236 "volcano plot" (- $\log _{10}\left(p\right.$-value) vs $\log _{2}$ (fold change)) for all the yeast genes in response

237 to $\mathrm{NaCl}$ in the $\mathrm{S}$ lines and the ancestral strain. Figure $3 \mathrm{~b}$ shows the extent of expression

238 change in the evolved lines (vertical axis) and in the ancestral strain (horizontal axis) for

239 all genes with similar response to $\mathrm{NaCl}$ in the evolved lines and the ancestral strain

240 ( $\mid \log _{2}$ (fold change) $\mid \geq 1$, t-test, $p$-value $<0.05$, False Discovery Rate $<10 \%$ ). The genes we

241 labeled by name in the figure include some known stress response genes (e.g., GPD1,

242 SIP18), some genes whose expression changed substantially (e.g., FMP48, NOG1),

243 and genes in both categories (e.g., GRE1). Many of these shared genes are directly

244 associated with saline stress, hyperosmotic stress, or the general stress response, and

245 were shown to be affected by hyper-osmolarity and salt stress in previous studies of the

246 physiological and osmotic stress response (Gasch et al., 2000; Posas et al., 2000; Rep

247 et al., 2000; Causton et al., 2001; Yale \& Bohnert, 2001). Among the 581 shared

248 induced genes, 192 genes were shown to be affected by stress in previous studies;

249 among the 580 shared repressed genes, 56 were affected by stress in previous studies.

$250 \quad$ Next, we classified shared genes using the Comprehensive Yeast Genome

251 Database (CYGD) classification from the Munich Information Center for Protein

252 Sequences (MIPS) (Güldener et al., 2005). A detailed analysis is given in the

253 supplementary material (supplementary figure 2 and supplementary text). Here, we only

254 discuss two gene classes in more detail. First, genes associated with 'cell rescue,

255 defense and virulence' contain many general stress response genes, as well as genes

256 that respond specifically to saline stress. One would expect that such genes are

257 induced in response to salt stress, and our data show that induced genes in this

258 category are overrepresented, and repressed genes in this category are

259 underrepresented (supplementary figure 2 and supplementary text). This group of

260 genes was also found to be significantly overrepresented among genes whose deletion

261 reduced the growth rate of yeast in salt (Warringer et al., 2003). A second class of

262 genes are genes involved in protein synthesis. The physiological response to stress can

263 cause repression of protein synthesis (Gasch et al., 2000). In support of this

264 observation, our data show that significantly fewer genes associated with protein 
265 synthesis are induced, and significantly more genes than expected are repressed

266 (supplementary figure 2 and supplementary text). Again, this group of genes was

267 significantly overrepresented among genes whose deletion increased salt-resistance

268 (Warringer et al., 2003).

269

272 strains is instructive, we were more interested in genes that are expressed differently.

273 These are genes whose expression adapted evolutionarily to salt stress. They can be

274 subdivided into two main categories. The first comprises genes whose regulation has

275 changed in the evolved lines. Figure $4 a$ and $b$ show two hypothetical examples of genes

276 in this category. The second category comprises genes whose basal level of expression

277 changed in the evolved lines, even in the absence of salt stress (Figure 4c and d). This

278 second category of genes may be especially important, because our selection

279 conditions imposed continuous salt stress. It is thus conceivable that cells whose

280 expression is ancestrally regulated in response to salt stress simply increase or

281 decrease their basal expression to the level of regulated expression in the ancestral

282 strain in the absence of salt. We note that these two categories have multiple

283 subcategories, and there can be genes where both the basal expression and regulation

284 can change. Supplementary figure 3 shows an overview of all possibilities.

$285 \quad$ We distinguished genes in the main categories by calculating two different Z-

286 scores for each gene, a $Z$-score for change in basal expression $\left(Z_{b}\right)$ and a $Z$-score for

287 change in regulation $\left(Z_{r}\right)$ (see Methods) (Mukhopadhyay et al., 2006). In this analysis,

288 we considered genes with |Z-score| $\geq 1.5$ as differentially expressed.

289

\section{Genes whose regulation changes}

$291 \quad$ In all, there are 62 genes whose regulation changes during our experiment

292 (Figure 5a). As we mentioned above, multiple types of such change are possible.

293 Firstly, a gene's induction in salt can increase. This will occur if the gene is induced

294 more strongly in the evolved lines than the ancestral strain (supplementary figure 3b).

295 Secondly, a gene's induction in salt can become reduced (supplementary figure 3c).

296 Thirdly, a gene's repression in salt can be increased, i.e., the gene becomes repressed

297 to a greater extent in the evolved lines (supplementary figure 3d). Fourth, a gene's

298 repression in salt can be reduced (supplementary figure 3e). Finally, a gene that was

299 not regulated in the ancestral strain can become regulated in response to salt

300 (supplementary figure $3 \mathrm{f}$ and $\mathrm{g}$ ). Figure $5 \mathrm{a}$ plots the extent to which genes changed in

301 their regulation against their $Z_{r}$ scores. Some of the genes with significant changes in

302 the regulation are labeled. Figure $6 a$ and $b$ show the five genes whose induction or

303 repression changed most significantly (based on $Z_{r}$ ). We will discuss some of them

304 further below.

$305 \quad$ There are only four genes in total which showed increased induction in the

306 evolved lines. Among these four genes, two of them (PUT4 and PCL5) also showed

307 decrease in basal expression in the evolved lines. Four further genes showed new

308 induction in the evolved lines. One of them, CUP1-1 (Figure 5a), has previously been 
309 observed to be up-regulated in response to osmotic stress (Yale \& Bohnert, 2001). Five

310 genes showed reduced induction in our experiment. One of the genes, HSP30, encodes

311 a stress-responsive protein that negatively regulates the $\mathrm{H}(+)$-ATPase Pma1p. It is

312 induced by heat shock, treatment with organic acid and ethanol, and glucose starvation

313 (Panaretou \& Piper, 1992; Piper et al., 1994; Piper et al., 1997).

$314 \quad$ In contrast to the few genes which showed increased induction, a total of 37

315 genes showed increased repression. Out of these 37 genes, one gene (HLR1) is

316 directly involved in the stress response. This gene encodes a protein involved in

317 regulation of cell wall composition, as well as in the osmotic stress response (Alonso-

318 Monge et al., 2001). 12 genes showed new repression in our experiment. Among these

31912 genes, the genes GCV2 and GCV1 were previously found to be up-regulated in

320 osmotic stress (Yale \& Bohnert, 2001). We did not find any gene with reduced

321 repression.

322 We next classified genes whose regulation changed in any of these ways

323 according to their functions, using the CYGD classification of yeast genes (Güldener et

324 al., 2005) (Figure 7), and compared their distribution among functional categories with

325 the proportions of the yeast genome in each category. The distribution of the number of

326 genes among different classes is non-random for genes whose repression changes (49

327 genes in total; Chi-square test, $p$-value $<0.001, d f=17)$, whereas the distribution is not

328 significantly different from random for the genes whose induction changes (13 genes; $p$ -

329 value $>0.1, \mathrm{df}=17$ ). We then asked whether genes whose regulation changes occur

330 preferentially in specific functional categories. Interestingly, for genes with change in

331 induction level, such genes are (marginally) enriched only in the functional class of cell

332 rescue, defense and virulence $(p=0.0444)$. Genes whose repression changes are

333 enriched in the functional classes transcription $(p=0.0006)$, protein synthesis

$334(p=0.0008)$, and proteins with binding or catalytic function $(p=0.0003)$. The classes

335 protein fate (folding, modification, destination; $p=0.0232$ ), cellular transport $(p=0.0048)$,

336 cell cycle/DNA processing $(p=0.0134)$ and cell rescue, defense and virulence

$337(p=0.0230)$ contain fewer genes whose repression chages than expected by chance

338 alone (all $p$-values based on an exact binomial test).

339

340 Genes with basal level expression change

$341 \quad$ Next we turn to genes that show a change in basal expression. There are 82

342 such genes. Thirty eight of them increased their basal expression, whereas 44 genes

343 reduced their basal expression. Figure $5 \mathrm{~b}$ plots the extent of change in basal expression

$344\left(\log _{2}\right.$-transformed) of all the genes against their corresponding $Z_{b}$ values. Some of the

345 genes with significant changes in the basal expression level are labeled. Figure $6 \mathrm{c}$ and

$346 \mathrm{~d}$ lists the top five genes (based on $Z_{b}$ ) with increased and decreased basal expression,

347 respectively. The distribution of genes among different functional classes (Figure 7) is

348 significantly different from what would be expected by chance alone for the genes with

349 increase in basal expression $(p<0.05, d f=17$, Chi-square test), and also for the genes

350 with a decrease in basal expression $(p<0.001, d f=17)$. For genes with an increase in

351 basal expression, the class 'cell rescue and defense' (stress response) shows

352 significantly more genes than expected ( $p=0.0059$, exact binomial test), and the class 
353 protein fate $(p=0.0409)$ shows significantly fewer genes than expected by chance alone.

354 For genes with a decrease in basal expression, the class 'cell type differentiation' (cell

355 wall, sporulation, spore wall etc.) $(p=0.0165)$ shows more genes than expected, and the

356 classes 'transcription' ( $p=0.0125$ ) as well as 'protein fate' (folding, modification,

357 destination) $(p=0.0014)$ contain fewer genes than expected.

$358 \quad$ Two well-established stress response genes showed an increase in basal

359 expression in the evolved lines. One of them is CTT1, whose basal expression

360 increased by $\sim 1.6$ fold compared to the ancestral strain. This gene was found to be

361 induced in four previous genome-wide studies of physiological stress adaptation in

362 yeast (Gasch et al., 2000; Rep et al., 2000; Causton et al., 2001; Yale \& Bonhert, 2001).

363 The second known stress response genes is the transcription factor MSN4, whose

364 expression was increased by approximately 1.5 fold. A more detailed analysis of

365 individual genes can be found in the supplementary material (supplementary text).

366

367 Whole Genome (Re)Sequencing Analysis

368 Some laboratory evolution studies have identified few beneficial mutations of

369 large fitness effects in evolving populations (Blanc \& Adams, 2003; Velicer et al., 2006;

370 Stanek et al., 2009). To find out if there are any such mutations, we subjected the

371 ancestral strain and population samples from two of our evolved lines (S1 and S2) to

372 deep sequencing at about $\sim 10 \mathrm{X}$ coverage and the third line (S3) to $\sim 50 \mathrm{X}$ coverage,

373 using the Roche 454 Genome Sequencer. In our analysis of this sequence data, we

374 aimed to identify only changes that may have swept to high frequency or fixation and

375 10X coverage is sufficient for that purpose. We chose candidate SNPs based on the

376 criteria for the changed (derived) nucleotide described in supplementary methods and

377 our approach is deliberately conservative. We identified 56 candidate SNPs and 7

378 deletions in total from all the three evolved (S) lines.

379 We sequenced all 56 candidate SNPs and 7 candidate deletions, but only

380 one of them turned out to be a true change. This change was a SNP unique to line S2,

381 and occurred at a frequency exceeding 50 percent, based both on next generation

382 sequencing data and PCR sequencing data. This SNP is a non-synonymous $G$ to $A$

383 mutation (amino acid change: G230D) in the MOT2 (YER068W) gene. The gene MOT2

384 is a subunit of the CCR4-NOT complex which has roles in transcription regulation,

385 mRNA degradation, and post-transcriptional modifications (Cade \& Errede, 1994; Liu et

386 al., 1998; Badarinarayana et al., 2000; Denis et al., 2001; Panasenko et al., 2006;

387 Mersman et al., 2009), with no known role in salt tolerance. To test whether the MOT2

388 SNP alone provided any fitness benefit to yeast in salt, we replaced the wild-type

389 variant of MOT2 in the genome of the ancestral strain with the mutant (see Methods).

390 Three replicate competition assays using FACS (see Methods) showed a relative

391 population fitness $w=1.043 \pm 0.008$ for the mutant MOT2 allele (Figure $8 a$ ) in medium

392 with $\mathrm{NaCl}$, compared to $w=0.999 \pm 0.014$ for the wildtype MOT2 allele. However, the

393 fitness increase of the mutant MOT2 could only explain 25.3 percent of the total amount

394 of fitness increase in line $S 2$, whose relative fitness was $w=1.17$, indicating that there

395 are other factors that contribute to the fitness increase of the line S2. In addition, we

396 note that the MOT2 mutation also conferred a substantial fitness increase in the 
397 absence of $\mathrm{NaCl}$ (Figure 8b), indicating that this mutation is not specifically adaptive to

398 salt stress.

399

400 Duplication and PFGE

$401 \quad$ We next turned to copy number variations as sources of evolutionary

402 adaptation. Since large-scale chromosomal rearrangements and aneuploidies are

403 frequent in yeast laboratory evolution (Adams et al., 1992; Dunham et al., 2002; Rancati

404 et al., 2008), and are also observed in yeast gene knock-out strains (Hughes et al.,

405 2000), we first wanted to know whether such rearrangements were numerous in our

406 evolved lines. To this end, we first performed pulsed field gel electrophoresis (PFGE)

407 analysis of whole chromosomes for two clones of the ancestral strain, as well as for two

408 clones from each of the evolved lines. This experiment revealed no changes in any of

409 the lines, except for one additional band (at $\sim 500 \mathrm{~kb}$ ) that occurred exclusively in line S3

410 (Figure 9a). PFGE for chromosomes digested with the Notl restriction enzyme also

411 revealed a single novel band $(\sim 180 \mathrm{~kb})$, which was again unique to line S3 (Figure 9b).

412 Taken together, these observations suggest that high frequency copy number changes

413 on a chromosomal scale are not rampant in our evolved lines, and the same

414 arrangements do not occur across lines. All other distinguishable fragments in the gel,

415 apart from the novel band of size $\sim 180 \mathrm{~kb}$ in line S3, were as predicted for a haploid

416 yeast genome from a computational restriction digest of the genome with Notl.

417 Additionally, chromosome-wide read coverage data from next generation sequencing

418 (see below) supports the notion that there are no copy number changes involving large

419 chromosome fragments (supplementary figure 4). However, a change in ploidy in the

420 evolved lines that would affect entire chromosomes is not detectable by any of these

421 two methods.

422

423 Gene Amplifications and Deletions from Next Generation Sequencing data

$424 \quad$ We next turned to smaller scale copy number changes that we could detect

425 in our next generation sequencing data. To detect gene duplication and deletions, a

426 segmentation algorithm (see supplementary methods) was used along with analysis of

427 DNA breakpoints from the reads (see supplementary Methods). The results of both

428 analyses are described in detail in the supplementary text.

$429 \quad$ We did not observe any large amplification or deletion in the genomes of any

430 of the lines (and specifically in line S3) which could explain the appearance of novel

431 bands in the pulsed field electrophoresis data from Figure 9. This observation, together

432 with read coverage data of individual chromosomes (Supplementary figure 4) suggests

433 that these novel bands were results of a non-duplicative translocation event in the line

434 S3. However, for a haploid genome, a translocation event would displace one of the

435 original bands in the PFGE, which we did not observe. Thus, such a translocation event

436 is only consistent with the PFGE data if the affected chromosome occurs in more than

437 one copy. In other words, it would be consistent with a ploidy change in line S3.

438

439 Ploidy of the Evolved Yeast Lines

$440 \quad$ Changes in ploidy of haploid yeast strains under salt stress have been 
441 observed before (Gerstein et al., 2006). Neither PFGE nor chromosome wide read 442 coverage data would be able to detect such changes. To estimate the ploidy level of 443 evolved lines in comparison to the ancestral strain, we grew ancestral strain and 444 population samples of the evolved lines for 24 hours at $30^{\circ} \mathrm{C}$ and estimated the cell 445 densities. We then isolated genomic DNA from a defined number of cells and quantified 446 the DNA amount, which allowed us to calculate the DNA content per yeast cell for all 447 the lines. We found that the DNA content per cell of lines S1 and S2 had increased by 44878.7 percent and 85.4 percent, respectively, from that of the ancestral strain. For the 449 line S3, the DNA content per cell was more than double that of the ancestral strain 450 (119.91 percent increase from the ancestral strain)(Figure 10). These observations 451 suggest that all the evolved lines have become massively aneuploid.

452

453 A consistent cell size increase during our experiment

454 Increases in ploidy are often associated with cell size increases. Our lines are no 455 exception, as exemplified by the histogram of Figure 11a, which shows the distribution 456 of cell diameters of the ancestral strain and the evolved line S2. This increase is also 457 microscopically visible (Figure 11b). The mean cell diameter increased from $2.35 \mu \mathrm{m}$ $458( \pm 0.70 \mu \mathrm{m})$ to $6.73 \mu \mathrm{m}( \pm 2.34 \mu \mathrm{m})$. Along with a significant increase in the mean cell 459 diameter (t-test, $p$-value $<2.2 \times 10^{-16}$ ), the coefficient of variation $\left(C_{v}\right)$, as defined by the 460 ratio of standard deviation and mean, also increased significantly (t-test for distributions 461 of $\mathrm{C}_{\mathrm{v}}$ for samples drawn from two distributions, $\mathrm{p}$-value $<10^{-6}$ ). In other words, not only 462 did cells become larger during laboratory evolution, they also became more variable in 463 size. Cell size changes in laboratory evolution experiments are not unprecedented. 464 They have been observed in evolving E. coli populations (Lenski \& Travisano, 1994; 465 Lenski \& Mongold, 2000; Philippe et al., 2009), as well as in Staphylococcus aureus 466 populations in $\mathrm{NaCl}$ medium (Vijaranakul et al., 1995). However, effect of cell size on 467 the survival and fitness are poorly understood.

468

469 Discussion

$470 \quad$ To investigate evolutionary adaptation to long-term osmotic stress, we

471 evolved yeast cells in the laboratory for 300 generations in high salt $(\mathrm{NaCl})$ medium. We

472 observed that salt reduces the growth rate of our ancestral strain by 11 percent.

473 Consequently, the final cell density after 24 hours of growth is approximately 3 times

474 lower compared to cells grown in the same medium but without salt. Our three replicate 475 evolved lines grow approximately 8 to 12 percent faster than the ancestral strain in high 476 salt medium, and part of this increase reflects adaptation specific to salt.

$477 \quad$ We analyzed the gene expression levels in all the evolved lines as well as in 478 the ancestral strain. Although there were many shared genes between the evolved lines 479 and the ancestral strain that respond to salt in similar manner, we also observed 480 multiple differentially expressed genes in the evolved lines compared to the ancestral 481 strain. These differentially expressed genes can be divided into two categories. The first 482 category comprises genes with changes in their basal expression level in the evolved 483 lines compared to the ancestor (i.e., even in the absence of salt). The second category 484 comprises genes regulated differently in response to salt in the evolved lines. 
491 studies show limited comparability with respect to the identity of these genes.

495 Both these genes were also up-regulated in response to salt in our ancestral strain.

496 CTT1 encodes for a cytosolic catalase and protects the cell from oxidative damage by

497 reactive oxygen species (Jamieson, 1998; Lushchak \& Gospodaryov, 2005; Herrero et

498 al., 2008). This gene has been shown to be up-regulated in four previous studies of

499 physiological stress response and salt stress response (Gasch et al., 2000; Rep et al.,

500 2000; Causton et al., 2001; Yale \& Bohnert, 2001). The gene GAC1 is associated with

501 glycogen synthesis (Wu et al., 2001) and is also induced during osmo-adaptation

502 (Posas et al., 2000). Both these genes contain stress responsive elements (STRE) in

503 their promoters (Schuller et al., 1994; Moskvina et al., 1998) and are known to be

504 activated under various stress conditions. A third gene, CUP1-1, showed increased

505 induction in the evolved lines and also was up-regulated in response to $\mathrm{NaCl}$ in our

506 ancestral strain. This gene was also shown to be up-regulated in one previous study of

507 physiological stress response (Yale \& Bohnert, 2001). Yet another gene, HLR1, showed

508 increased repression in the evolved lines, and was down-regulated in the ancestral

509 strain in $\mathrm{NaCl}$ medium. This gene encodes a protein involved in maintaining cell wall

510 composition and is also involved in response to osmotic stress (Alonso-Monge et al.,

$5112001)$.

$512 \quad$ There are three main physiological components of the salt stress response.

513 They affect adaptation time to salt, growth rate in salt, and efficiency of growth in salt

514 (Warringer et al., 2003). In our experiments, evolutionary adaptation to salt must affect

515 one or more of these three component, because the viability itself of yeast cells does

516 not change between the ancestral strain and the evolved lines. There are various ways

517 in which these components could change during evolution. Firstly, some of the genes

518 whose basal expression changes might be directly involved in the initial adaptation to

519 salt. Increasing or decreasing the basal expression of those genes to the level needed

520 for salt adaptation, might enable the cells respond to salt much more quickly as

521 opposed to changing their expression state. For example, some ion transporter genes

522 (e.g. FRE5) have an increased basal expression in the evolved lines in our experiment.

523 Higher levels of these transporter proteins at the initial stages of the salt stress

524 response would help cells achieve ion homeostasis much more quickly. Secondly, some

525 of the differentially expressed genes might not be directly related to the salt stress

526 response but encode transcription factors that controls salt stress response genes. For

527 example, the transcription factor MSN4, that controls many stress response genes, has

528 increased basal expression levels in our evolved yeast lines. Higher levels of this 
529 transcription factor during the initial adaptation phase could ensure faster induction of

530 the genes required for salt stress response. Thirdly, there are genes that show

531 evolutionary change in regulation in the evolved lines. Higher level of induction or

532 repression of these genes might increase the growth rate and/or efficiency of growth in

533 salt medium.

$534 \quad$ We found 16 genes that gained regulation (new induction and new

535 repression) in the evolution experiment, suggesting that new ways of salt stress

536 adaptation could also arise during evolution. However, we also observed loss of

537 regulation, at least to some extent, for five genes (genes with reduced induction and

538 reduced repression) in the evolved lines. Because we performed our evolution

539 experiment under constant environmental stress, losing regulation might be

540 advantageous for evolutionary adaptation to salt stress. If the affected genes are

541 involved in adaptation to multiple stresses, losing regulation could actually be beneficial

542 both under constant or fluctuating environmental stressors. However, if the affected

543 genes are specific for that particular stress, a loss in regulation may also have a cost,

544 because cells would become physiologically less flexible under fluctuating

545 environmental stresses. Among the five genes with loss of regulation, one gene,

546 HSP30, can be induced by several stresses (Piper et al., 1997). However, the mRNA

547 level of this gene was shown to be diminished in $\mathrm{NaCl}$ medium by Rep et al (Rep et al.,

548 2000).

$549 \quad$ The hyperosmotic and salt stress responses are complex and influenced by

550 many genes. It is an open question whether a small number of genetic changes with

551 large effects could dramatically increase salt stress resistance, and thus explain the

552 fitness increase in our evolved lines. To find out, we also sequenced the ancestral strain

553 and population samples of the evolved lines using deep sequencing with genome

554 coverages between 10 - and 50 -fold to identify whether any high frequency

555 polymorphisms arose in the evolved lines during our evolution experiment. For two

556 reasons, we chose deep population sequencing over clone sequencing. First,

557 population sequencing gives a comprehensive view of polymorphisms in a population.

558 Second, it can also permit estimation of polymorphism frequencies, although any such

559 estimate would be more qualitative than quantitative at our sequence coverage. At the

560 very least, population sequencing can detect high frequency polymorphisms. Such

561 polymorphisms are of the greatest interest to us, because they would correspond to

562 adaptive mutations with strong fitness effects that rose to high frequencies. We note

563 that the population sizes in our experiment are so large that no neutral polymorphisms

564 would be expected to rise to high frequencies during the experiment's short duration

565 except through hitchhiking with an advantageous mutation (effective population size in

566 our experiment is $>2 \times 10^{6}$ ). (Clone sequencing can detect low frequency polymorphisms,

567 but for a heterogeneous population, this method requires sequencing of many clones,

568 and is thus currently prohibitively costly.)

$569 \quad$ Only one of our evolved lines (S2) contained a high frequency single

570 nucleotide polymorphism (SNP). This SNP occurs in more than 50 percent of the

571 population, and causes an amino acid substitution in the protein encoded by the MOT2

572 gene. When introduced into the ancestral strain, this mutation causes a fitness increase 
573 that explains 25.3 percent of the increase in fitness $w$ in the evolved strain. However,

574 the mutation causes a fitness increase also in the absence of salt, which means that its

575 effects do not reflect a specific adaptation to salt.

$576 \quad$ Multiple genetic changes of low population frequency could be present in our

577 evolved lines, and these changes might explain a part of the fitness increase we see.

578 Such low frequency SNPs might be present for several reasons. First, individual

579 mutations may confer only modest fitness benefits and thus increase in frequency

580 slowly. Second, some mutations with very strong fitness effects may have occurred, but

581 late in the experiment, and thus might simply not have had enough time to rise to high

582 frequency.. Third, the fitness effect of alleles might be determined by epistatic

583 interactions with other mutations elsewhere in the genome (Elena \& Lenski, 1997; Elena

584 \& Lenski, 2001; Morgan \& Feldman, 2001). Eventually genotypes with such alleles

585 might rise to high frequency, but at time scales much longer than those of our

586 experiment, because multiple mutations might have to occur before any one epistatic

587 combination with strong benefits arises. Finally, and perhaps most importantly, there

588 could be clonal interference between multiple beneficial polymorphisms in the

589 population and this might prevent any particular polymorphism from rising to high

590 frequency in the population (Kao \& Sherlock, 2008).

591 Similar to our analysis on SNPs, our analysis on copy number variations,

592 both based on PFGE and next generation sequencing, did not reveal any large scale

593 copy number changes shared across strains. However, we observed ploidy increases in

594 all the evolved lines, and this could be the reason behind increase in cell size of these

595 lines.

$596 \quad$ Evidence from plants suggests that an increase in ploidy and the resultant

597 increase in cell size can be advantageous under salt-stressed conditions. For example,

598 within a species, plants with higher ploidy can cope with salt stress better than plants

599 with lower ploidy (Saleh et al., 2008). Polyploid plants have higher water content and

600 lower osmotic pressure than diploid plant (Noggle, 1946). Their increased water content

601 is due to a decrease in the surface-to-volume ratio of cells (Stebbins, 1950). In addition,

602 any water content decrease due to high salinity is smaller in polyploid plants than that in

603 diploid plants (Tal \& Gardi, 1976). Gerstein et al. observed that initially haploid yeast

604 lines increased genome size significantly faster under salt stress than in unstressed

605 condition, suggesting an advantage for higher ploidy or increased cell size also in yeast

606 (Gerstein et al., 2006).

$607 \quad$ Changes in ploidy could help yeast cells adapt faster physiologically, for

608 example by affecting expression of genes important for salt stress tolerance. Could all

609 the changes in gene expression that we observed in our experiment be caused solely

610 by ploidy changes or by the concomitant increase in cell size? To find out, we compared

611 the set of genes that changed expression in our evolved lines with the set of genes that

612 are known to change expression after cell size increases (Wu et al., 2010) and ploidy

613 changes (Galitski et al., 1999). Remarkably, only three genes that evolve changed

614 expression in our experiment (out of 144 differentially expressed genes in our dataset)

615 are among the genes affected by cell size increase or ploidy changes. Two of these

616 genes showed increased basal expression in our experiment. One of them, YIL169C, 
617 was also observed to be induced with increase in cell size (Wu et al., 2010). The other 618 gene, COS8, was observed to change expression with a change in ploidy level (Galitski 619 et al., 1999). The third gene, YLR042C, showed a basal decrease in expression in our 620 evolved lines. It was also observed to be repressed in cells with increased size (Wu et 621 al., 2010).

622 Overall, the vast majority of changes that we observe in gene expression 623 cannot be caused by changed ploidy or cell size. Because our whole genome 624 sequencing analysis revealed no small genetic changes of strong phenotypic effects, 625 the gene expression changes we observe could be caused by multiple genetic changes 626 of modest individual effects. Some of these changes might affect a gene's expression in $627 \mathrm{cis}$, others in trans, for example through changes in the amino acid sequence of 628 transcriptional regulators (Wittkopp et al., 2004; Emerson \& Li, 2010). In addition, some 629 expression changes may be caused by epigenetic change (e.g., changes in DNA 630 methylation) or alterations in cellular memory (Turner, 2002; Ringrose \& Paro, 2004; 631 Zhang et al., 2005; Zacharioudakis et al., 2007).

$632 \quad$ We found several genes with modest changes in expression level in our 633 evolved lines, and no gene with very strong expression change. Our observations stand 634 in contrast to the only laboratory evolution study we know of that investigated

635 evolutionary adaptation to a stressor. The study asked how E. coli cells adapt to heat 636 stress. It observed very high level of evolutionary expression change in two proteins, 637 GroEL and GroES, that are known heat shock genes (Rudolph et al., 2010). The reason 638 for this difference to our work is probably that adaptation to salt stress requires several 639 molecular functions simultaneously, namely ionic detoxification to achieve ionic 640 equilibrium, maintaining cellular water activity, and osmolyte synthesis. Each of the 641 evolutionarily altered genes in our experiment may perform or control some of these 642 functions and thus, may have only a modest individual fitness contribution when 643 differentially expressed. In addition, gene interactions (Phillips 2008; He et al., 2010) 644 could also contribute towards fitness increase. This scenario resembles recent 645 observations in genome wide association studies (GWAS) for complex human traits and 646 diseases, where researchers have observed many genetic variants with modest effects 647 on a phenotype (Frayling, 2007; Barrett et al., 2008; Cooper et al., 2008; Visscher, 648 2008; Zeggini et al., 2008; Hindorff et al., 2009; Manolio et al., 2009; Visscher \& 649 Montgomery, 2009; Park et al., 2010; Yang et al., 2010).

$650 \quad$ Taken together, adaptation to salt stress is associated with DNA content 651 increase and gene expression change in all the three lines, one high frequency mutation 652 in one of the evolved lines, and one chromosomal rearrangement in another. The 653 increase in DNA content in all the lines is massive and previous studies suggest that the 654 change in ploidy might be beneficial for the lines in salt. In contrast, there are 144 genes 655 in our study that show modest change in their expression levels. This observation 656 suggests that tinkering with expression levels of several genes might be a useful 657 mechanism to respond to a complex, polygenic trait rather than altering expression of 658 few genes to very high levels. Such tinkering might also reflect cellular constraints on 659 evolution. For example, changing expression of a gene to very high levels or 660 suppressing a gene completely might increase fitness of the yeast cells in salt medium. 
661 However, changing expression of a gene to very high levels or suppressing a gene 662 completely might affect the capability of the cells to grow in other media. Since we do 663 not find high frequency genetic changes that can explain expression changes of the 664 genes, it is possible that these are caused by multiple genetic changes of low frequency 665 (that might vary from one subpopulation to other) or by epigenetic changes. These 666 results also suggest that cells in our populations perhaps employ several strategies in 667 combination for adaptation to salt stress rather than following a single path to

668 adaptation. It is possible that such genetic heterogeneity might render the populations to 669 be more robust in the long term. If some of the genetic factors contributing to the salt 670 stress adaptation are lost, the fitness of the population would not fall drastically. Also, 671 such diverse populations might be less vulnerable against other environmental

672 fluctuations which might co-occur with saline stress in natural environment. Although

673 individual contributions of each of these genetic mechanisms to adaptation remain

674 unclear, our study provides a stepping stone for asking further questions on

675 evolutionary adaptation of yeast to salt stress.

676

\section{Methods}

678 Strains and media.

679 All laboratory evolution experiments started from the same clone of haploid yeast strain 680 BY4741, which is referred to as the ancestral strain. The 3 replicate yeast lines evolved 681 in $\mathrm{NaCl}$ are referred to as lines S1, S2 and S3. The growth rates of the evolved lines as 682 well as the ancestral strain were measured relative to a BY4741 strain in which the 683 CWP2 gene was GFP-tagged (termed as the reference strain). For serial transfers, cells 684 were cultured in YP and $2 \%$ galactose (YPG) and YPG supplemented with $0.5 \mathrm{M} \mathrm{NaCl}$ 685 (YPGN).

686

\section{Serial transfer}

688 Six parallel serial transfer experiments were started from one single clone of the 689 ancestral strain. In each parallel experiment, $50 \mathrm{ml}$ yeast culture was grown for at $30^{\circ} \mathrm{C}$. 690 Every 24 hours, $50 \mu \mathrm{l}$ of grown culture was transferred into fresh culture medium; 30

691 such transfer cycles were carried out for a total of approximately 300 generations (Each 692 transfer cycle involved approximately $\log _{2} 1000 \approx 10$ cell generations). In three of the 693 parallel experiments, the culture medium was YPG, whereas in the other three parallel 694 experiments, the medium was YPGN.

695

\section{Viability assays}

697 To estimate cell viabilities, cultures of growing yeast cells were sampled after 16 hours 698 (during exponential growth phase), as well as after 24 hours (during stationary phase), 699 diluted and plated. The plates were incubated at $30^{\circ} \mathrm{C}$ for 5 days and the number of 700 colonies were counted. The relative viabilities of both the ancestral strain and the 701 evolved lines were estimated from the ratio of colonies forming on agar plates

702 containing YPG $+0.5 \mathrm{M} \mathrm{NaCl}$ to that of plates containing only YPG. All the 703 measurements were carried out in three biological replicates. 


\section{Competition assays}

706 To compare growth rates of the evolved lines with that of the ancestral strain,

707 competition assays were carried out in triplicates using fluorescence activated cell

708 sorting (FACS). Cells from frozen glycerol stocks were grown overnight in $4 \mathrm{ml}$ YPD

709 medium $\left(30^{\circ} \mathrm{C}, 220 \mathrm{rpm}\right)$ until late logarithmic phase $\left(<1.5 \times 10^{8} \mathrm{cell} / \mathrm{sl}\right)$. For the

710 competition assay, approximately equal cell numbers of the reference strain and of the

711 competing strain were mixed and grown for 24 hours at $30^{\circ} \mathrm{C}$. The relative cell numbers

712 at the beginning and at the end of the competition experiment were determined using

713 FACS (supplementary methods) and growth rate differences were estimated as

714 described in supplementary methods. For calculation of fitness, the relative cell

715 numbers at the beginning of the assay were taken into consideration.

716

717 Whole genome transcriptome analysis

718 The mRNA expression levels in the ancestral strain and in the evolved lines were

719 analyzed using a GeneChip Yeast Genome 2.0 Array (Affymetrix). Equal number of

720 cells from the ancestral strain and the evolved lines were grown in YPG medium for 16

721 hours. The cells were then either induced with $0.5 \mathrm{M} \mathrm{NaCl}$ or grown uninduced for 20

722 further minutes. The microarray analysis was carried out for 2 replicates each for the

723 ancestral strain in YPG and YPGN (4 in total) and for 4 replicate population samples (1

724 for S1,2 for S2 and 1 for S3) each in YPG and YPGN for the S lines (8 in total).

725 "Shared" genes, genes that respond to $\mathrm{NaCl}$ in a similar manner between the ancestral

726 strain and the evolved lines, with significant up-regulation or down-regulation were

727 identified using a t-test at $p=0.05$, False Discovery Rate $(F D R)<10 \%$ and $\mid \log _{2}$ (fold

728 change) $\mid \geq 1$.

$729 \quad$ Genes with changes in basal expression or change in regulation were

730 identified based on two $Z$ scores $Z_{r}$ and $Z_{b}$ (see supplementary methods). Genes whose

731 absolute $Z$-scores exceeded a value of 1.5 were considered to be differentially

732 expressed. The differentially expressed genes were then grouped into different classes

733 using the CYGD functional classification for yeast genes (Güldener et al., 2005).

734

735 Whole Genome Sequencing and SNP identification

736 The ancestral strain, as well as population samples of the $\mathrm{NaCl}$ evolved lines at

737 generation 300 were sequenced at approximately 10X coverage using next generation

738 pyrosequencing (Margulies et al., 2005) (genome sequencer FLX LR system, Roche).

739 The line S3 was further sequenced to a total of $\sim 50 \mathrm{X}$ coverage. Candidate SNPs and

740 indels were identified using blastn (Altschul et al., 1990) followed by MUSCLE (Edgar,

7412004 ) based on the criteria described in supplementary methods. PCR sequencing was

742 done to confirm candidate SNPs and indels.

743

744 Pulsed-field gel electrophoresis (PFGE)

745 To identify large scale chromosomal rearrangements, two clones from each of the

746 evolved lines (S1, S2 and S3) and the ancestral strain were analyzed by PFGE.

747 Agarose plugs were prepared with the CHEF Yeast Genomic DNA Plug Kit (Bio-Rad)

748 according to the manufacturer's protocol. For restriction enzyme digestion, agarose 
749 plugs were incubated with Notl restriction enzyme for $16 \mathrm{hrs}$ at $37^{\circ} \mathrm{C}$ mixture in an

750 appropriate restriction buffer. The digested or undigested plugs were then loaded into

751 the wells of $1 \%$ agarose gels. Electrophoresis was carried out in a CHEF-DRIII system

752 (Bio-Rad). The gels were stained using ethidium bromide (EtBr) and photographed.

753

754 Gene Amplification and Deletions from Next Generation Sequencing data

$755 \quad$ In addition to identifying very short indels within individual reads, larger gene

756 duplications and deletions in the evolved lines, compared to the ancestral strain, were

757 identified using read coverage information from the sequencing data. Furthermore, DNA

758 breakpoint analysis was performed to confirm those candidate copy number changes.

759

760 Ploidy determination of the evolved lines

761 To estimate the ploidy level of the evolved lines in comparison to the

762 ancestral strain, the ancestral strain and population samples of the evolved lines were

763 grown for 24 hours at $30^{\circ} \mathrm{C}$. Genomic DNA was isolated from a defined number of cells

764 following the protocol described in (Sambrook \& Russell, 2001, Chapter 6).

765

766 Cell size measurement

767 Population samples of ancestral and evolved cells were grown in YPG medium up to

768 mid-log phase. The cell density was adjusted to be the same for all the samples

$769\left(\sim 1 \times 10^{7} \mathrm{cells} / \mathrm{ml}\right)$. 20ul of cells were then loaded onto disposable counting chambers and

770 the cell sizes, were measured using a Cellometer M10 (Nexcelom Biosciences),

771 following the manufacturer's protocol.

772

773 Acknowledgements

774 We would like to acknowledge the help of the Functional Genomics Center Zurich for

775 carrying out next generation sequencing and microarray experiments. AW

776 acknowledges support through SNF grants 315200-116814, 315200-119697, and

777 315230-129708, as well as through the YeastX project of SystemsX.ch, and the

778 University Research Priority Program in systems biology at the University of Zurich.

\section{References}

780 Adams, J. \& Oeller, P.W. 1986. Structure of evolving populations of Saccharomyces

781 cerevisiae: Adaptive changes are frequently associated with sequence alterations

782 involving mobile elements belonging to the Ty family. Proc. Natl. Acad. Sci. USA 83:

783 7124-7127.

784

785 Adams, J., Puskas-Rozsa, S., Simlar, J. \& Wilke, C.M. 1992. Adaptation and major

786 chromosomal changes in populations of Saccharomyces cerevisiae. Curr. Genet. 22:

787 13-19.

788

789 Alonso-Monge, R., Real, E., Wojda, I., Bebelman, J.P., Mager, W.H. \& Siderius, M.

790 2001. Hyperosmotic stress response and regulation of cell wall integrity in

791 Saccharomyces cerevisiae share common functional aspects. Mol. Microbiol. 41: 717- 
792730.

793

794 Altschul, S.F., Gish, W., Miller, W., Myers, E.W. \& Lipman, D.J. 1990. Basic local

795 alignment search tool. J. Mol. Biol. 215: 403-410.

796

797 Apse, M.P., Aharon, G.S., Snedden, W.A. \& Blumwald, E. 1999. Salt Tolerance

798 Conferred by Overexpression of a Vacuolar $\mathrm{Na}+/ \mathrm{H}+$ Antiport in Arabidopsis. Science

799 285: $1256-1258$.

800

801 Attfield, P.V. 1997. Stress Tolerance: The key to effective strains of industrial baker's 802 yeast. Nat. Biotech. 15: 1351-1357.

803

804 Badarinarayana, V., Chiang, Y.C. \& Denis, C.L. 2000. Functional interaction of CCR4-

805 NOT proteins with TATAA-binding protein (TBP) and its associated factors in yeast.

806 Genetics 155: 1045-1054.

807

808 Barrett, J.C., Hansoul, S., Nicolae, D.L., Cho, J.H., Duerr, R.H., Rioux, J.D. et al. 2008.

809 Genome-wide association defines more than 30 distinct susceptibility loci for Crohn's

810 disease. Nat. Genet. 40: 955-962.

811

812 Betz, C., Zajonc, D., Moll, M. \& Schweizer, E. 2002. ISC1-encoded inositol

813 phosphosphingolipid phospholipase $\mathrm{C}$ is involved in $\mathrm{Na}+/ \mathrm{Li}+$ halotolerance of

814 Saccharomyces cerevisiae. Eur. J. Biochem. 269: 4033-4039.

815

816 Blanc, V.M. \& Adams, J. 2003. Evolution in Saccharomyces cerevisiae: Identification of 817 Mutations Increasing Fitness in Laboratory Populations. Genetics 165: 975-983.

818

819 Bressan, R.A., Hasegawa, P.M. \& Pardo J.M. 1998. Plants use calcium to resolve salt

820 stress. Trends Plant Sci. 3: 411-412.

821

822 Brewster, J.L., de Valoir, T., Dwyer, N.D., Winter, E. \& Gustin, M.C. 1993. An

823 Osmosensing Signal Transduction Pathway in Yeast. Science 259: 1760-1763.

824

825 Cade, R.M. \& Errede, B. 1994. MOT2 encodes a negative regulator of gene expression

826 that affects basal expression of pheromone-responsive genes in Saccharomyces

827 cerevisiae. Mol. Cell. Biol. 14: 3139-3149.

828

829 Causton, H.C., Ren, B., Koh, S.S., Harbison, C.T., Kanin, E., Jennings, E.G. et al. 2001.

830 Remodeling of Yeast Genome Expression in Response to Environmental Changes. Mol.

831 Biol. Cell 2: 323-337.

832

833 Cooper, J.A. 1994. MAP KINASE PATHWAYS: Straight and narrow or tortuous and 834 intersecting? Curr. Biol. 4: 1118-1121.

835 
836 Cooper, J.D., Smyth, D.J., Smiles, A.M., Plagnol, V., Walker, N.M., Allen, J.E. et al.

837 2008. Meta-analysis of genome-wide association study data identifies additional type 1

838 diabetes risk loci. Nat. Genet. 40: 1399-1401.

839

840 Denis, C.L., Chiang, Y.C., Cui, Y. \& Chen, J. 2001. Genetic evidence supports a role for 841 the yeast CCR4-NOT complex in transcriptional elongation. Genetics 158: 627-634.

842

843 Dihazi, H., Kessler, R. \& Eschrich, K. 2004. High Osmolarity Glycerol (HOG) Pathway-

844 induced Phosphorylation and Activation of 6-Phosphofructo-2-kinase Are Essential for

845 Glycerol Accumulation and Yeast Cell Proliferation under Hyperosmotic Stress. J. Biol.

846 Chem. 279: 23961-23968.

847

848 Dunham, M.J., Badrane, H., Ferea, T., Adams, J., Brown, P.O., Rosenzweig, F. et al.

849 2002. Characteristic genome rearrangements in experimental evolution of

850 Saccharomyces cerevisiae. Proc. Natl. Acad. Sci. USA 99: 16144-16149.

851

852 Edgar, R.C. 2004. MUSCLE: multiple sequence alignment with high accuracy and high

853 throughput. Nucleic Acids Res. 32:1792-1797.

854

855 Elena, S.F. \& Lenski, R.E. 1997. Test of synergistic interactions among deleterious

856 mutations in bacteria. Nature 390: 395-398.

857

858 Elena, S.F. \& Lenski, R.E. 2001. Epistasis between new mutations and genetic

859 background and a test of genetic canalization. Evolution 55: 1746-1752.

860

861 Emerson, J.J. \& Li, W-H. 2010. The genetic basis of evolutionary change in gene

862 expression levels. Phil. Trans. R. Soc. B 365: 2581-2590.

863

864 Errede, B. \& Levin, D.E. 1993. A conserved kinase cascade for MAP kinase activation

865 in yeast. Curr. Opin. Cell Biol. 5: 254-260.

866

867 Espinosa-Ruiz, A., Belles, J.M., Serrano, R. \& Culianez-Macla, V. 1999. Arabidopsis

868 thaliana AtHAL3: A flavoprotein related to salt and osmotic tolerance and plant growth.

869 Plant J. 20: 529-539.

870

871 Ferea, T.L., Botstein, D., Brown, P.O. \& Rosenzweig, R.F. 1999. Systematic changes in

872 gene expression patterns following adaptive evolution in yeast. Proc. Natl. Acad. Sci.

873 USA. 96: 9721-9726.

874

875 Ferrando, A., Kron, S.J., Rios, G., Fink, G.R. \& Serrano, R. 1995. Regulation of cation

876 transport in Saccharomyces cerevisiae by the salt tolerance gene HAL3. Mol. Cell. Biol.

877 15: 5470-5481.

878

879 Frayling, T.M. 2007. Genome-wide association studies provide new insights into type 2 
880 diabetes aetiology. Nat. Rev. Genet. 8: 657-662.

881

882 Galcheva-Gargova, Z., Derijard, B., Wu, I.H. \& Davis, R.J. 1994. An osmosensing signal

883 transduction pathway in mammalian cells. Science 265: 806-808.

884

885 Galitski, T., Saldanha, A.J., Styles, C.A., Lander, E.S. \& Fink, G.R. 1999. Ploidy

886 regulation of gene expression. Science 285: 251-254.

887

888 Ganster, R.W., McCartney, R.R. \& Schmidt, M.C. 1998. Identification of a Calcineurin889 Independent Pathway Required for Sodium Ion Stress Response in Saccharomyces 890 cerevisiae. Genetics 150: 31-42.

891

892 Garciadeblas, B., Rubio, F., Quintero, F.J., Bañuelos, M.A., Haro, R. \& Rodríguez-

893 Navarro, A. 1993. Differential expression of two genes encoding isoforms of the ATPase 894 involved in sodium efflux in Saccharomyces cerevisiae. Mol. Gen. Genet. 236: 363-368. 895

896 Gasch, A.P., Spellman, P.T., Kao, C.M., Carmel-Harel, O., Eisen, M.B., Storz, G. et al. 897 2000. Genomic Expression Programs in the Response of Yeast Cells to Environmental 898 Changes. Mol. Biol. Cell 11: 4241-4257.

899

900 Gaxiola, R., de Larrinoa, I.F., Villalba, J.M. \& Serrano, R. 1992. A novel and conserved 901 salt-induced protein is an important determinant of salt tolerance in yeast. EMBO J.11: 902 3157-3164.

903

904 Gaxiola, R.A., Rao, R., Sherman, A., Grisafi, P., Alper, S.L. \& Fink, G.R. 1999. The 905 Arabidopsis thaliana proton transporters, AtNhx1 and Avp1, can function in cation 906 detoxification in yeast. Proc. Natl. Acad. Sci. USA 96: 1480-1485.

907

908 Gerstein, A.C., Chun, H.J.E., Grant, A. \& Otto, S.P. 2006. Genomic convergence toward 909 diploidy in Saccharomyces cerevisiae. PLoS Genet. 2: e145.

910

911 Gisbert, C., Rus, A.M., Bolarín, M.C., Lopez-Coronado, J.M., Arrillaga, I., Montesinos,

912 C. et al. 2000. The Yeast HAL1 Gene Improves Salt Tolerance of Transgenic Tomato.

913 Plant Physiol. 123: 393-402.

914

915 Goossens, A., Forment, J. \& Serrano, R. 2002. Involvement of Nst1p/YNL091w and

$916 \mathrm{Ms} 11 \mathrm{p}$, a U2B" splicing factor, in Saccharomyces cerevisiae salt tolerance. Yeast 19:

917 193-202.

918

919 Gorner, W., Durchschlag, E., Martinez-Pastor, M.T., Estruch, F., Ammerer, G. \&

920 Hamilton, B. et al. 1998. Nuclear localization of the $\mathrm{C} 2 \mathrm{H} 2$ zinc finger protein Msn2p is

921 regulated by stress and protein kinase A activity. Genes Dev. 12: 586-597.

922

923 Gresham, D., Desai, M.M., Tucker, C.M., Jenq, H.T., Pai, D.A., Ward, A. et al. 2008. 
924 The Repertoire and Dynamics of Evolutionary Adaptations to Controlled Nutrient-

925 Limited Environments in Yeast. PLoS Genet. 4: e1000303.

926

927 Güldener, U., Münsterkötter, M., Kastenmüller, G., Strack, N., van Helden, J., Lemer, C.

928 et al. 2005. CYGD: the Comprehensive Yeast Genome Database. Nucl. Acids Res. 33:

929 D364-D368.

930

931 Han, J., Lee, J.D., Bibbs, L. \& Ulevitch, R.J. 1994. A MAP kinase targeted by endotoxin

932 and hyperosmolarity in mammalian cells. Science 265: 808-811.

933

934 Haro, R., Garciadeblas, B. \& Rodríguez-Navarro, A. 1991. A novel P-type ATPase from 935 yeast involved in sodium transport. FEBS Lett. 291: 189-191.

936

937 Hasegawa, P.M. \& Bressan, R.A. 2000. Plant Cellular and Molecular Responses to

938 High Salinity. Annu. Rev. Plant. Physiol. Plant. Mol. Biol. 51: 463-499.

939

$940 \mathrm{He}$, X., Qian, W., Wang, Z., Li, Y. \& Zhang, J. 2010. Prevalent positive epistasis in

941 Escherichia coli and Saccharomyces cerevisiae metabolic networks. Nat. Genet. 42

942 272-276.

943

944 Heath, V.L., Shaw, S.L., Roy, S. \& Cyert, M.S. 2004. Hph1p and Hph2p, Novel

945 Components of Calcineurin-Mediated Stress Responses in Saccharomyces cerevisiae.

946 Eukaryotic Cell 3: 695-704

947

948 Herrero, E., Ros, J., Bellí, G. \& Cabiscol, E. 2008. Redox control and oxidative stress in 949 yeast cells. Biochim. Biophys. Acta 1780: 1217-1235.

950

951 Hindorff, L.A., Sethupathy, P., Junkins, H.A., Ramos, E.M., Mehta, J.P., Collins, F.S. et

952 al. 2009. Potential etiologic and functional implications of genome-wide association loci

953 for human diseases and traits. Proc. Natl. Acad. Sci. USA 106: 9362-9367.

954

955 Hirata, Y., Andoh, T., Asahara, T. \& Kikuchi, A. 2003. Yeast Glycogen Synthase Kinase9563 Activates Msn2p-dependent Transcription of Stress Responsive Genes. Mol. Biol.

957 Cell. 14: 302-312.

958

959 Hirt, H. 1997. Multiple roles of MAP kinases in plant signal transduction. Trends Plant

960 Sci. 2: 11-15.

961

962 Hohmann, S. 2002. Osmotic Stress Signaling and Osmoadaptation in Yeasts. Micro.

963 Mol. Biol. Rev. 66: 300-372.

964

965 Hughes, T.R., Roberts, C.J., Dai, H., Jones, A.R., Meyer, M.R., Slade, D. et al. 2000.

966 Widespread aneuploidy revealed by DNA microarray expression profiling. Nat. Genet.

967 25: 333-337. 
969 Jamieson, D.J. 1998. Oxidative stress responses of the yeast Saccharomyces

970 cerevisiae. Yeast 14: 1511-1527.

971

972 Jonak, C., Kiegerl, S., Ligterink, W., Barker, P.J., Huskisson, N.S. \& Hirt, H. 1996.

973 Stress signaling in plants: A mitogen-activated protein kinase pathway is activated by

974 cold and drought. Proc. Natl. Acad. Sci. USA 93: 11274-11279.

975

976 Jonak, C., Ligterink, W. \& Hirt, H. 1999. MAP kinases in plant signal transduction. Cell.

977 Mol. Life Sci. 55: 204-213.

978

979 Kao, K.C. \& Sherlock, G. 2008. Molecular characterization of clonal interference during

980 adaptive evolution in asexual populations of Saccharomyces cerevisiae. Nat. Genet. 40:

981 1499-1504.

982

983 Kosako, H., Nishida, E. \& Gotoh, Y. 1993. cDNA cloning of MAP kinase kinase reveals 984 kinase cascade pathways in yeasts to vertebrates. EMBO J. 12: 787-794.

985

986 Kovtun, Y., Chiu, W-L., Tena, G., Sheen, J. 2000. Functional analysis of oxidative

987 stress-activated mitogen-activated protein kinase cascade in plants. Proc. Natl. Acad.

988 Sci. USA 97: 2940-2945.

989

990 Kyriakis, J.M. \& Avruch, J. 2001. Mammalian Mitogen-Activated Protein Kinase Signal

991 Transduction Pathways Activated by Stress and Inflammation. Physiol. Rev. 81: 807-

992869.

993

994 Lee, J.H., Montagu, M.V. \& Verbruggen, N. 1999. A highly conserved kinase is an 995 essential component for stress tolerance in yeast and plant cells. Proc. Natl. Acad. Sci. 996 USA 96: 5873-5877.

997

998 Lenski, R.E. \& Travisano, M. 1994. Dynamics of adaptation and diversification: A 999 10,000-generation experiment with bacterial populations. Proc. Natl. Acad. Sci. USA 91: 1000 6808-6814.

1001

1002 Lenski, R.E. \& Mongold, J.A. 2000. Cell size, shape, and fitness in evolving populations

1003 of bacteria. In: Scaling in Biology (J. H. Brown \& G. B. West, eds), pp. 221-235, Oxford 1004 University Press.

1005

1006 Liu, H.Y., Badarinarayana, V., Audino, D.C., Rappsilber, J., Mann, M. \& Denis, C.L.

1007 1998. The NOT proteins are part of the CCR4 transcriptional complex and affect gene

1008 expression both positively and negatively. EMBO J. 17: 1096-1106.

1009

1010 Lushchak, V.I. \& Gospodaryov, D.V. 2005. Catalases protect cellular proteins from 1011 oxidative modification in Saccharomyces cerevisiae. Cell Biol. Int. 29: 187-192. 
1013 Maathuis, F.J.M. \& Amtmann, A. 1999. K+ Nutrition and Na+ Toxicity : The Basis of

1014 Cellular K+/Na+ Ratios. Annals of Bot. 84: 123-133.

1015

1016 Mai, B. \& Breeden, L. 1997. Xbp1, a Stress-Induced Transcriptional Repressor of the

1017 Saccharomyces cerevisiae Swi4/Mbp1 Family. Mol. Cell. Biol. 17: 6491-6501.

1018

1019 Manolio, T.A., Collins, F.S., Cox, N.J., Goldstein, D.B., Hindorff, L.A., Hunter, D.J. et al.

1020 2009. Finding the missing heritability of complex diseases. Nature 461: 747-753.

1021

1022 Margulies, M., Egholm, M., Altman, W.E., Attiya, S., Bader, J.S., Bemben, L.A. et al.

1023 2005. Genome sequencing in microfabricated high-density picolitre reactors. Nature

1024 437: 376-380.

1025

1026 Martinez-Pastor, M.T., Marchler, G., Schuller, C., Marchler-Bauer, A., Ruis, H. \&

1027 Estruch, F. 1996. The Saccharomyces cerevisiae zinc finger proteins Msn2p and Msn4p

1028 are required for transcriptional induction through the stress-response element (STRE).

1029 EMBO J. 15: 2227-2235.

1030

1031 Mendizabal, I., Rios, G., Mulet, J.M., Serrano, R. \& de Larrinoa, I.F. 1998. Yeast

1032 putative transcription factors involved in salt tolerance. FEBS Lett. 425: 323-328.

1033

1034 Mendoza, I., Rubioli, F., Rodriguez-Navarroli, A. \& Pardo, J.M. 1994. The Protein

1035 Phosphatase Calcineurin Is Essential for $\mathrm{NaCl}$ Tolerance of Saccharomyces cerevisiae.

1036 J. Biol. Chem. 269: 8792-8796.

1037

1038 Mersman, D.P., Du, H.N., Fingerman, I.M., South, P.F. \& Briggs, S.D. 2009.

1039 Polyubiquitination of the demethylase Jhd2 controls histone methylation and gene

1040 expression. Genes Dev. 23: 951-962.

1041

1042 Morgan, L.W. \& Feldman, J.F. 2001. Epistatic and Synergistic Interactions Between

1043 Circadian Clock Mutations in Neurospora crassa. Genetics 159: 537-543.

1044

1045 Moskvina, E., Schuller, C., Maurer, C.T.C., Mager, W.H. \& Ruis, H. 1998. A Search in

1046 the Genome of Saccharomyces cerevisiae for Genes Regulated via Stress Response

1047 Elements. Yeast 14: 1041-1050.

1048

1049 Mulet, J.M., Leube, M.P., Kron, S.J., Rios, G., Fink, G.R. \& Serrano, R. 1999. A novel 1050 mechanism of ion homeostasis and salt tolerance in yeast: the Hal4 and Hal5 protein

1051 kinases modulate the Trk1-Trk2 potassium transporter. Mol. Cell. Biol. 19: 3328-3337.

1052

1053 Mukhopadhyay, A., He, Z., Alm, E.J., Arkin, A.P., Baidoo, E.E., Borglin, S.C. et al. 2006:

1054 Salt Stress in Desulfovibrio vulgaris Hildenborough: an Integrated Genomics Approach.

1055 J. Bacteriol. 188: 4068-4078. 
1057 Murguía, J.R., Bellés, J.M. \& Serrano, R. 1996. The yeast HAL2 nucleotidase is an in

1058 vivo target of salt toxicity. J. Biol. Chem. 271: 29029-29033.

1059

1060 Neiman, A.M. 1993. Conservation and reiteration of a kinase cascade. Trends Genet. 9:

1061 390-394.

1062

1063 Neiman, A.M., Stevenson, B.J., Xu, H-P., Sprague, Jr. G.F., Herskowitz, I., Wigler, M. et

1064 al. 1993. Functional Homology of Protein Kinases Required for Sexual Differentiation in

1065 Schizosaccharomyces pombe and Saccharomyces cerevisiae Suggests a Conserved

1066 Signal Transduction Module in Eukaryotic Organisms. Mol. Biol. Cell 4: 107-120.

1067

1068 Noggle, R. 1946.The physiology of polyploidy in plants. I. Review of the literature.

1069 Lloydia 9; 153-173.

1070

1071 Nishida, E. \& Gotoh, Y. 1993. The MAP kinase cascade is essential for diverse signal

1072 transduction pathways. Trends Biochem. Sci. 18: 128-131.

1073

1074 Ostrander, D.B. \& Gorman, J.A. 1999. The Extracellular Domain of the Saccharomyces

1075 cerevisiae SIn1p Membrane Osmolarity Sensor Is Necessary for Kinase Activity. J.

1076 Bacteriol. 181: 2527-2534.

1077

1078 Panaretou, B. \& Piper, P.W. 1992. The plasma membrane of yeast acquires a novel

1079 heat-shock protein (hsp30) and displays a decline in proton-pumping ATPase levels in

1080 response to both heat shock and the entry to stationary phase. Eur. J. Biochem. 206:

$1081635-640$.

1082

1083 Panasenko, O., Landrieux, E., Feuermann, M., Finka, A., Paquet, N. \& Collart, M.A.

1084 2006. The yeast Ccr4-Not complex controls ubiquitination of the nascent-associated

1085 polypeptide (NAC-EGD) complex. J. Biol. Chem. 281: 31389-31398.

1086

1087 Pardo, J.M., Reddy, M.P., Yang, S., Maggio, A., Huh, G-H., Matsumoto, T. et al. 1998.

1088 Stress signaling through $\mathrm{Ca}^{2+} /$ calmodulin-dependent protein phosphatase calcineurin

1089 mediates salt adaptation in plants. Proc. Natl. Acad. Sci. USA 95: 9681-9686.

1090

1091 Park, J.H., Wacholder, S., Gail, M.H., Peters, U., Jacobs, K.B., Chanock, S.J. et al.

1092 2010. Estimation of effect size distribution from genome-wide association studies and 1093 implications for future discoveries. Nat. Genet. 42: 570-575.

1094

1095 Pelech, S.L. \& Sanghera, J.S. 1992. Mitogen-activated protein kinases: versatile

1096 transducers for cell signaling. Trends Biochem. Sci. 17: 233-238.

1097

1098 Philippe, N., Pelosi, L., Lenski, R.E. \& Schneider, D. 2009. Evolution of Penicillin-

1099 Binding Protein 2 Concentration and Cell Shape during a Long-Term Experiment with 
1100 Escherichia coli. J. Bacteriol. 191: 909-921.

1101

1102 Phillips, P.C. 2008. Epistasis - the essential role of gene interactions in the structure

1103 and evolution of genetic systems. Nat. Rev. Genet. 9: 855-867.

1104

1105 Piper, P.W., Ortiz-Calderon, C., Holyoak, C., Coote, P. \& Cole, M. 1997. Hsp30, the

1106 integral plasma membrane heat shock protein of Saccharomyces cerevisiae, is a stress-

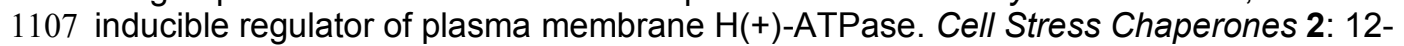

110824.

1109

1110 Piper, P.W., Talreja, K., Panaretou, B., Moradas-Ferreira, P., Byrne, K., Praekelt, U.M.

1111 et al. 1994. Induction of major heat-shock proteins of Saccharomyces cerevisiae,

1112 including plasma membrane Hsp30, by ethanol levels above a critical threshold.

1113 Microbiology 140: 3031-3038.

1114

1115 Posas, F., Chambers, J.R., Heyman, J.A., Hoeffler, J.P., de Nadal, E. \& Arino, J. 2000.

1116 The Transcriptional Response of Yeast to Saline Stress. J. Biol. Chem. 275: 17249-

111717255.

1118

1119 Quinteroa, F.J., Blatt, M.R. \& Pardo, J.M. 2000. Functional conservation between yeast 1120 and plant endosomal $\mathrm{Na}+/ \mathrm{H}+$ antiporters. FEBS Lett. 471: 224-228.

1121

1122 Quintero, F.J., Ohta, M., Shi, H., Zhu, J-K. \& Pardo, J.M. 2002. Reconstitution in yeast

1123 of the Arabidopsis SOS signaling pathway for $\mathrm{Na}^{+}$homeostasis. Proc. Natl. Acad. Sci.

1124 USA 99: 9061-9066.

1125

1126 Rancati, G., Pavelka, N., Fleharty, B., Noll, A., Trimble, R., Walton, K. et al. 2008.

1127 Aneuploidy underlies rapid adaptive evolution of yeast cells deprived of a conserved

1128 cytokinesis motor. Cell 135: 879-893.

1129

1130 Reiser, V., Salah, S.M. \& Ammerer, G. 2000. Polarized localization of yeast Pbs2

1131 depends on osmostress, the membrane protein Sho1 and Cdc42. Nat. Cell. Biol. 2: 620-

1132627.

1133

1134 Rep, M., Reiser, V., Gartner, U., Thevelein, J.M., Hohmann, S., Ammerer, G. et al.

1135 1999. Osmotic Stress-Induced Gene Expression in Saccharomyces cerevisiae Requires 1136 Msn1p and the Novel Nuclear Factor Hot1p. Mol. Cell. Biol. 19: 5474-5485.

1137

1138 Rep, M., Krantz, M., Thevelein, J.M. \& Hohmann, S. 2000. The transcriptional response

1139 of Saccharomyces cerevisiae to osmotic shock. J. Biol. Chem. 275: 8290-8300.

1140

1141 Ringrose, L. \& Paro, R. 2004. Epigenetic regulation of cellular memory by the Polycomb 1142 and Trithorax group proteins. Annu. Rev. Genet. 38:413-443.

1143 
1144 Rios, G., Ferrando, A. \& Serrano, R. 1997. Mechanisms of salt tolerance conferred by 1145 overexpression of the HAL1 gene in Saccharomyces cerevisiae. Yeast 13: 515-528.

1146

1147 Rudolph, B., Gebendorfer, K.M., Buchner, J. \& Winter, J. 2010. Evolution of Escherichia

1148 coli for Growth at High Temperatures. J. Biol. Chem. 285: 19029-19034.

1149

1150 Saito, H. \& Tatebayashi, K. 2004. Regulation of the Osmoregulatory HOG MAPK

1151 Cascade in Yeast. J. Biochem. 136: 267-272.

1152

1153 Saleh, B., Allario, T., Dambier, D., Ollitrault, P. \& Morillon, R. 2008. Tetraploid citrus

1154 rootstocks are more tolerant to salt stress than diploid. C. R. Biologies, 331: 703-710.

1155

1156 Samani, P. \& Bell, G. 2010. Adaptation of experimental yeast populations to stressful

1157 conditions in relation to population size. J. Evol. Biol. 23: 791-796.

1158

1159 Sambrook, J. \& Russell, D.W. 2001. Molecular Cloning: A laboratory Manual, 3rd edn.

1160 Cold Spring Harbor Laboratory Press, New York.

1161

1162 Sanchez, I., Hughes, R.T., Mayer, B.J., Yee, K., Woodgett, J.R. \& Avruch, J. et al. 1994.

1163 Role of SAPK/ERK kinase-1 in the stress-activated pathway regulating transcription

1164 factor c-Jun. Nature 372: 794-798.

1165

1166 Sanders, D. 2000. Plant biology: The salty tale of Arabidopsis. Curr. Biol. 10: R486-

1167 R488

1168

1169 Schmitt, A.P. \& McEntee, K. 1996. Msn2p, a zinc finger DNA-binding protein, is the

1170 transcriptional activator of the multistress response in Saccharomyces cerevisiae. Proc.

1171 Natl. Acad. Sci. USA 93: 5777-5782.

1172

1173 Schuller, C., Brewster, J.L., Alexander, M.R., Gustin, M.C. \& Ruis, H. 1994. The HOG

1174 pathway controls osmotic regulation of transcription via the stress response element

1175 (STRE) of the Saccharomyces cerevisiae CTT1 gene. EMBO J. 13: 4382-4389.

1176

1177 Serrano, R. 1996. Salt tolerance in plants and microorganisms: toxicity targets and

1178 defense responses. Int. Rev. Cytol. 165: 1-52.

1179

1180 Serrano, R., Mulet, J.M., Rios, G., Marquez, J.A., de Larrinoa, I.F., Leube, M.P. et al.

1181 1999. A glimpse of the mechanisms of ion homeostasis during salt stress. J. Exp. Bot.

1182 50: 1023-1036.

1183

1184 Stanek, M.T., Cooper, T.F. \& Lenski, R.E. 2009. Identification and dynamics of a

1185 beneficial mutation in a long-term evolution experiment with Escherichia coli. BMC Evol.

1186 Biol. 9:302.

1187 
1188 Stebbins, G. L. 1950. Variation and evolution in plants. Columbia University Press, New 1189 York.

1190

1191 Tal, M., \& Gardi, I. 1976. Physiology of polyploid plants: water balance in autotetraploid 1192 and diploid tomato under low and high salinity. Physiol. Plantarum 38: 257-261.

1193

1194 Teige, M., Scheikl, E., Eulgem, T., Doczi, R., Ichimura, K., Shinozaki, K. et al. 2004. The

1195 MKK2 Pathway Mediates Cold and Salt Stress Signaling in Arabidopsis. Mol. Cell 15:

1196 141-152.

1197

1198 Tsuda, L., Inoue, Y.H., Yoo, M.A., Mizuno, M., Hata, M., Lim, Y.M. et al. 1993. A protein 1199 kinase similar to MAP kinase activator acts downstream of the raf kinase in Drosophila.

1200 Cell 72: 407-414.

1201

1202 Tsujimoto, Y., Izawa, S. \& Inoue, Y. 2000. Cooperative Regulation of DOG2, Encoding

1203 2-Deoxyglucose-6-Phosphate Phosphatase, by Snf1 Kinase and the High-Osmolarity

1204 Glycerol-Mitogen-Activated Protein Kinase Cascade in Stress Responses of

1205 Saccharomyces cerevisiae. J. Bacteriol. 182: 5121-5126.

1206

1207 Trainotti, N. \& Stambuk, B. U. 2001. NaCl stress inhibits maltose fermentation by

1208 Saccharomyces cerevisiae. Biotech. Lett. 23: 1703-1707.

1209

1210 Turner, B.M. 2002. Cellular Memory and the Histone Code. Cell 111: 285-291.

1211

1212 Velicer, G.J., Raddatz, G., Keller, H., Deiss, S., Lanz, C., Dinkelacker, I. et al. 2006.

1213 Comprehensive mutation identification in an evolved bacterial cooperator and its

1214 cheating ancestor. Proc. Natl. Acad. Sci. USA 103: 8107-8112.

1215

1216 Vijaranakul, U., Nadakavukaren, M.J., de Jonge, B.L., Wilkinson, B.J. \& Jayaswal, R.K.

1217 1995. Increased Cell Size and Shortened Peptidoglycan Interpeptide Bridge of $\mathrm{NaCl}$ -

1218 Stressed Staphylococcus aureus and Their Reversal by Glycine Betaine. J. Bacteriol.

1219 177: 5116-5121.

1220

1221 Visscher, P.M. 2008. Sizing up human height variation. Nat. Genet. 40: 489-490.

1222

1223 Visscher, P.M. \& Montgomery, G.W. 2009. Genome-wide Association Studies and

1224 Human Disease: From Trickle to Flood. J. Am. Med. Assoc. 302: 2028-2029.

1225

1226 Warringer, J., Ericson, E., Fernandez, L., Nerman, O. \& Blomberg, A. 2003. High-

1227 resolution yeast phenomics resolves different physiological features in the saline

1228 response. Proc. Natl. Acad. Sci. USA 100: 15724-15729.

1229

1230 Waskiewicz, A.J. \& Cooper, J.A. 1995. Mitogen and stress response pathways: MAP

1231 kinase cascades and phosphatase regulation in mammals and yeast. Curr. Opin. Cell 
1232 Biol. 7:798-805.

1233

1234 Wittkopp, P.J., Haerum, B.K. \& Clark, A.G. 2004. Evolutionary changes in cis and trans

1235 gene regulation. Nature 430: 85-88.

1236

1237 Wu, C-Y., Rolfe, P.A., Gifford, D.K. \& Fink, G.R. 2010. Control of Transcription by Cell

1238 Size. PLoS Biol 8: e1000523.

1239

1240 Wu, X., Hart, H., Cheng, C., Roach, P.J. \& Tatchell, K. 2001. Characterization of Gac1p,

1241 a regulatory subunit of protein phosphatase type I involved in glycogen accumulation in

1242 Saccharomyces cerevisiae. Mol. Genet. Genomics 265: 622-635.

1243

1244 Yale, J. \& Bohnert, H.J. 2001. Transcript Expression in Saccharomyces cerevisiae at

1245 High Salinity. J. Biol. Chem. 276: 15996-16007.

1246

1247 Yan, M., Dai, T., Deak, J.C., Kyriakis, J.M., Zon, L.I., Woodgett, J.R. et al. 1994.

1248 Activation of stress-activated protein kinase by MEKKI phosphorylation of its activator

1249 SEKI. Nature 372: 798-800.

1250

1251 Yang, S.X., Zhao, Y.X., Zhang, Q., He, Y.K., Zhang, H. \& Luo, D. 2001. HAL1 mediate

1252 salt adaptation in Arabidopsis thaliana. Cell Res. 11: 142-148.

1253

1254 Yang, J., Benyamin, B., McEvoy, B.P., Gordon, S., Henders, A.K., Nyholt, D.R. et al.

1255 2010. Common SNPs explain a large proportion of the heritability for human height. Nat.

1256 Genet. 42: 565-569.

1257

1258 Yashar, B.M., Kelley, C., Yee, K., Errede, B. \& Zon, L.I. 1993. Novel Members of the

1259 Mitogen-Activated Protein Kinase Activator Family in Xenopus laevis. Mol. Cell. Biol. 13:

1260 5738-5748.

1261

1262 Zacharioudakis, I., Gligoris, T. \& Tzamarias, D. 2007. A Yeast Catabolic Enzyme

1263 Controls Transcriptional Memory. Curr. Biol. 17: 2041-2046.

1264

1265 Zamanillo, D., Hunt, T. \& Nebreda, A.R. 1994. A novel kinase cascade triggered by

1266 chemical stress and heat shock which stimulates MAP kinase-activated protein kinase-2

1267 and phosphorylation of the small heat shock proteins. Cell 78: 1027-1037.

1268

1269 Zeggini, E., Scott, L.J., Saxena, R., Voight, B.F., Marchini, J.L., Hu, T. et al. 2008. Meta-

1270 analysis of genome-wide association data and large-scale replication identifies

1271 additional susceptibility loci for type 2 diabetes. Nat. Genet. 40: 638-645.

1272

1273 Zeyl, C., Vanderford, T. \& Carter, M. 2003. An Evolutionary Advantage of Haploidy in

1274 Large Yeast Populations. Science 299: 555-558.

1275 
1276 Zhang, Y., Fatima, N. \& Dufau, M.L. 2005. Coordinated Changes in DNA Methylation

1277 and Histone Modifications Regulate Silencing/Derepression of Luteinizing Hormone

1278 Receptor Gene Transcription. Mol. Cell. Biol. 25: 7929-7939.

1279

1280 Zheng, D.Q., Wu, X.C., Tao, X.L., Wang, P.M., Li, P., Chi, X.Q., Li, Y.D., Yan, Q.F. \&

1281 Zhao, Y.H. 2011. Screening and construction of Saccharomyces cerevisiae strains with

1282 improved multi-tolerance and bioethanol fermentation performance. Bioresour. Technol.

1283 102:3020-3027.

1284

1285 Zhu, J-K. 2001. Plant salt tolerance. Trends Plant Sci. 6: 66-71.

1286

1287 Zhu, J-K. 2002. Salt and Drought Stress Signal Transduction in Plants. Annu. Rev.

1288 Plant. Biol. 53: 247-273.

1289

1290 Figures

1291 Figure 1 - (a-c) Viability of the ancestral strain and the evolved lines in medium

1292 containing $0.5 \mathrm{M} \mathrm{NaCl}$. Cells from the ancestral strain and the evolved lines from

1293 overnight cultures were transferred into YPG medium. Cell samples from these cultures

1294 were withdrawn after 16 hours (during exponential growth phase) as well as after 24

1295 hours (stationary phase), and then diluted and plated. The plates were incubated at

$129630^{\circ} \mathrm{C}$ for five days and the number of colonies were counted. The relative viability of

1297 both the ancestral strain and the evolved lines were estimated from the ratio of colonies

1298 formed on agar plates with YPGN medium to that of plates with YPG medium. Relative

1299 viabilities were measured for (a) the ancestral strain, (b) lines evolved in YPGN medium

1300 (S lines), and (c) lines evolved in YPG medium (C lines). From the figures, it is clear that

$1301 \mathrm{NaCl}$ does not affect the viability of the ancestral strain. The viabilities of the evolved

1302 lines S1, S2 and S3 do not increase during the course of adaptation.

1303

1304 Figure 2 - Fitness Assay of the evolved yeast lines. Fitness assays were performed

1305 by inoculating equal numbers of cells from an evolved line and from the GFP-tagged

1306 reference yeast strain in the same vessel. The cells were grown for $24 \mathrm{hrs}$, and the

1307 percentage of non-GFP-tagged cells was counted using FACS. The ratio of the cell

1308 numbers of the evolved lines to that of the ancestral strain gives the relative fitness of

1309 the evolved lines. (a) Relative fitness of the three replicate yeast lines evolved in

1310 medium with salt (S1,S2 and S3) in YPGN medium, and the three replicate yeast lines

1311 evolved without $\mathrm{NaCl}(\mathrm{C} 1, \mathrm{C} 2, \mathrm{C} 3)$ measured in YPGN medium. (b) Relative fitness of

1312 the lines $\mathrm{S} 1, \mathrm{~S} 2$ and $\mathrm{S} 3$ in medium without $\mathrm{NaCl}$. The lines evolved in salt show an

1313 increase in growth rate by $8 \%$ to $12 \%$ in $\mathrm{NaCl}$ medium compared to the ancestral strain.

1314 These lines also show a growth rate increase in medium without $\mathrm{NaCl}$. However, this

1315 increase in growth rate is consistently lower than the increase in $\mathrm{NaCl}$ medium. In

1316 addition, the lines evolved in medium without $\mathrm{NaCl}$ show lower fitness in $\mathrm{NaCl}$ medium.

1317 Taken together, it can be concluded that part of the adaptation in the salt evolved lines

1318 is specific to $\mathrm{NaCl}$.

1319 
1320 Figure 3 - The genes showing similar (t-test, p-value $<0.05$, False Discovery

1321 Rate $<10 \%$ ) induction or repression by $\mathrm{NaCl}$ in the ancestral strain and in the

1322 evolved $S$ lines (a) Volcano plot, showing the $p$-values on the $Y$ axis, and the fold

1323 expression change for these genes on the $X$ axis. The labeled genes include genes

1324 associated with the stress response (e.g., GPD1, SIP18), genes with high level of

1325 expression change (e.g., FMP48, NOG1), and genes in both categories (e.g., GRE1).

1326 (b) Scatter plot for the fold-change of the common genes in the ancestral strain (X-axis)

1327 and in the evolved $\mathrm{S}$ lines ( $\mathrm{Y}$-axis). Although most of the common genes change

1328 expression to a similar extent in the ancestral strain and the $\mathrm{S}$ lines, a small number of

1329 genes change expression to a different extent.

1331 Figure 4 - Schematic diagram for the type of expression change that can occur as

1332 a result of evolutionary adaptation in the evolved $\mathbf{S}$ lines compared to the

1333 ancestral strain. Each bar chart reflects the expression level of a hypothetical gene in

1334 the ancestral and evolved strain, in the absence and presence of salt. The scenarios

1335 that are depicted are as follows (a) No basal change, increased regulation (b) No basal

1336 change, increased repression (c) Basal increase in expression but no change in

1337 regulation (d) Basal decrease in expression but no change in regulation.

1338

1339 Figure 5 - Genes with differential expression in the $\mathbf{S}$ lines compared to the

1340 ancestral strain (|Z-score| $\geq 1.5)$. The plots show the level of expression change

1341 (vertical axis) and the Z-score (horizontal axis) for genes with (a) changed regulation,

1342 and (b) changed basal expression in the evolved lines. One should note that the basal

1343 change in expression is represented in terms of fold change, where the expression

1344 change is calculated as the ratio of the expressions in the evolved lines and the

1345 ancestral strain. The calculation of regulated expression change involves an additional

1346 subtraction of basal expression change and thus, can not be represented as fold

1347 change.

1348

1349 Figure 6 - The five genes with the highest Z-score in each of four categories of

1350 expression change in the evolved lines. Categories are (a) change in the level of

1351 induction, (b) change in the level of repression, (c) increase in basal expression, and (d)

1352 decrease in basal expression. The columns show, from left to right, the systematic gene

1353 name, the extent of expression change, the Z-score, the gene's common name, the

1354 type of expression change (in ( $a$ ) and b) only), and a reference, if the gene had

1355 responded physiologically to hyperosmotic stress or saline stress in previous studies.

1356

1357 Figure 7 - Different functional gene classes (Güldener et al., 2005) are enriched or

1358 impoverished for genes that change expression in the salt evolved yeast lines (S

1359 lines), compared to the ancestral strain. The vertical axis shows the functional

1360 classes of genes we consider, and the horizontal axis shows the fraction of genes in

1361 each category listed on the bottom of the figure. Asterisks ('*') indicate classes where

1362 the number of genes differs significantly from that expected by chance alone, as

1363 indicated by an exact binomial test. 
1365 Figure 8 - Fitness analysis (using FACS) of the ancestral strain into which the 1366 mutant MOT2 gene of line S2 was introduced, and the corresponding wild-type

1367 MOT2 gene as a control. (a) Fitness in medium with $\mathrm{NaCl}$ (b) Fitness in medium

1368 without $\mathrm{NaCl}$. The data show that the mutant MOT2 gene increases the fitness in salt

1369 medium as well as in medium without salt, and to similar extents.

1371 Figure 9 - Pulsed field gel electrophoresis (PFGE) analysis of the three replicate

1372 salt evolved lines S1,S2 \& S3 and the ancestral strain. Two clones from each of the

1373 lines S1,S2 and S3 were subjected to PFGE analysis along with the ancestral strain. (a)

1374 The PFGE for the yeast chromosomes from the evolved lines S1, S2 and S3 and the

1375 ancestral strain. The chromosomes are shown on the left side. M represents the yeast

1376 chromosome PFG marker (NEB Catalog\# N0345S). Only line S3 shows a new band

1377 (red arrow) of size approximately 500kb. (b) PFGE analysis of the Notl digested

1378 chromosomes from the lines S1,S2,S3 and the ancestral strain. M represents the Mid-

1379 range PFG marker I from NEB (Catalog\# N3551S), whose fragments range between 15

$1380 \mathrm{~kb}$ and $291 \mathrm{~kb}$. Again, only line S3 shows a new band (red arrow) in the gel, which has

1381 a size of approximately $180 \mathrm{~kb}$.

1382

1383 Figure 10 - DNA content per yeast cell of the ancestral strain and the evolved

1384 lines.

1385 The ancestral strain and population samples of the evolved lines were grown for 24

1386 hours at $30^{\circ} \mathrm{C}$. For each of the cultures, the cell density was estimated. Genomic DNA

1387 was isolated from a defined number of cells and DNA quantification was carried out with

1388 a NanoDrop ND-1000 spectrophotometer. The DNA content per cell was then

1389 calculated for each line. The figure suggests that all the evolved lines have become

1390 massively aneuploid.

1391

1392 Figure 11 - Cell size comparison between ancestral strain and one evolved line.

1393 (a) Histogram of cell diameters of the ancestral strain and the S2 line, showing that the

1394 salt evolved cells are bigger than the ancestral cells. (b) Microphotograph of cell

1395 samples from the ancestral strain and the salt evolved lines. 
1408
1409
1410
1411
1412
$141 \$$
1414
1415
1416
1417 Figure 1:
1418

1419

1420

1421

1422

1423

1424

1425

1426

1427

1428

1429

1430

1431

1432

1433

1434

1435

1436

1437

1438

1439

1440

1441

1442

1443

1444

1445

1446

1447

1448

1449

1450

1451

(a)

(c)

(b)
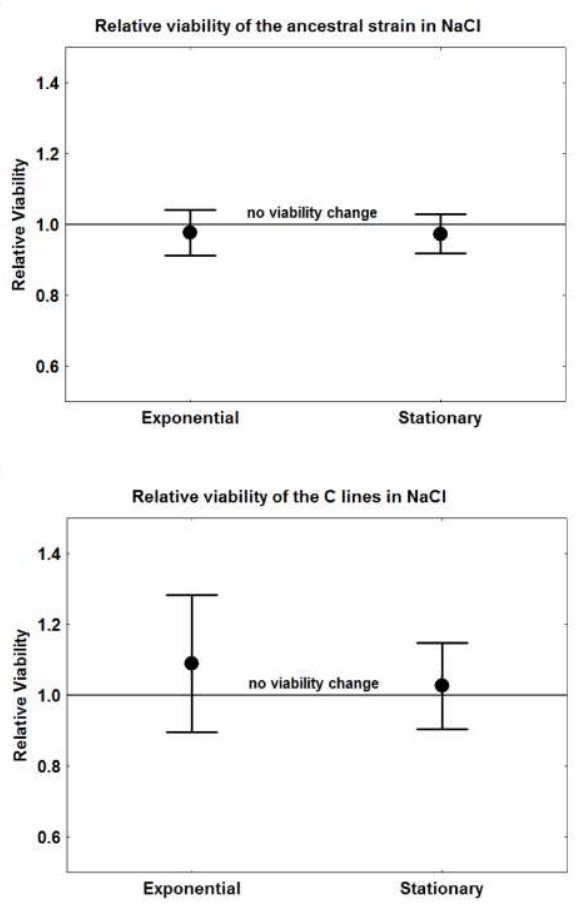

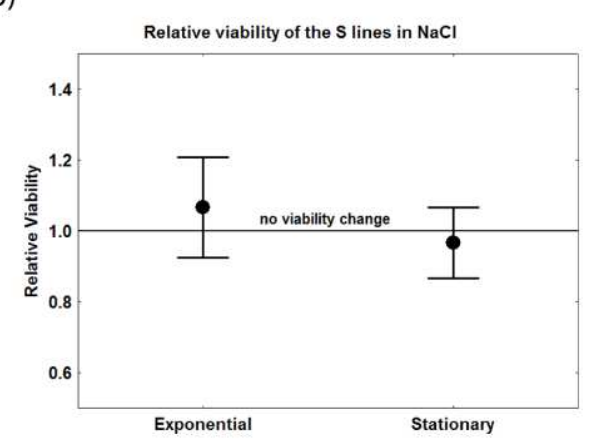


1454 Figure 2:

1455

1456

1457

1458

1459

1460

1461

1462

1463

1464

1465

1466

1467

1468

1469

1470

1471

1472

1473

1474

1475

1476

1477

1478

1479

1480

1481

1482

1483

1484

1485

1486

1487

1488

1489

1490

1491 Figure

1492 (a)

1493

1494

1495 (a)

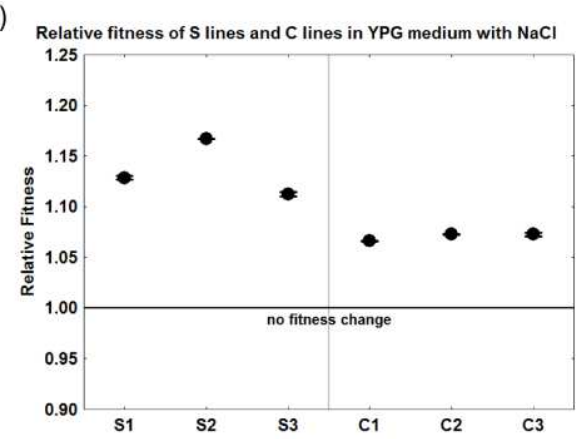

(b)

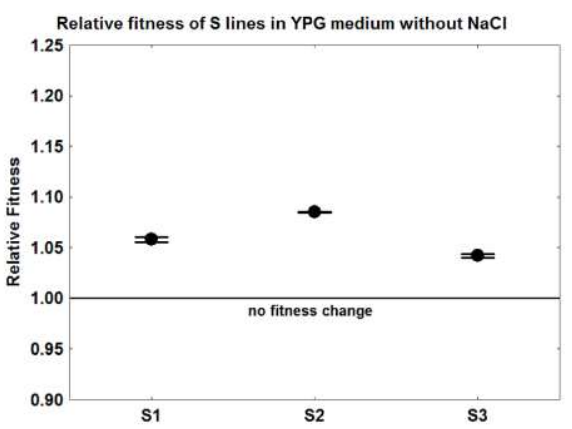

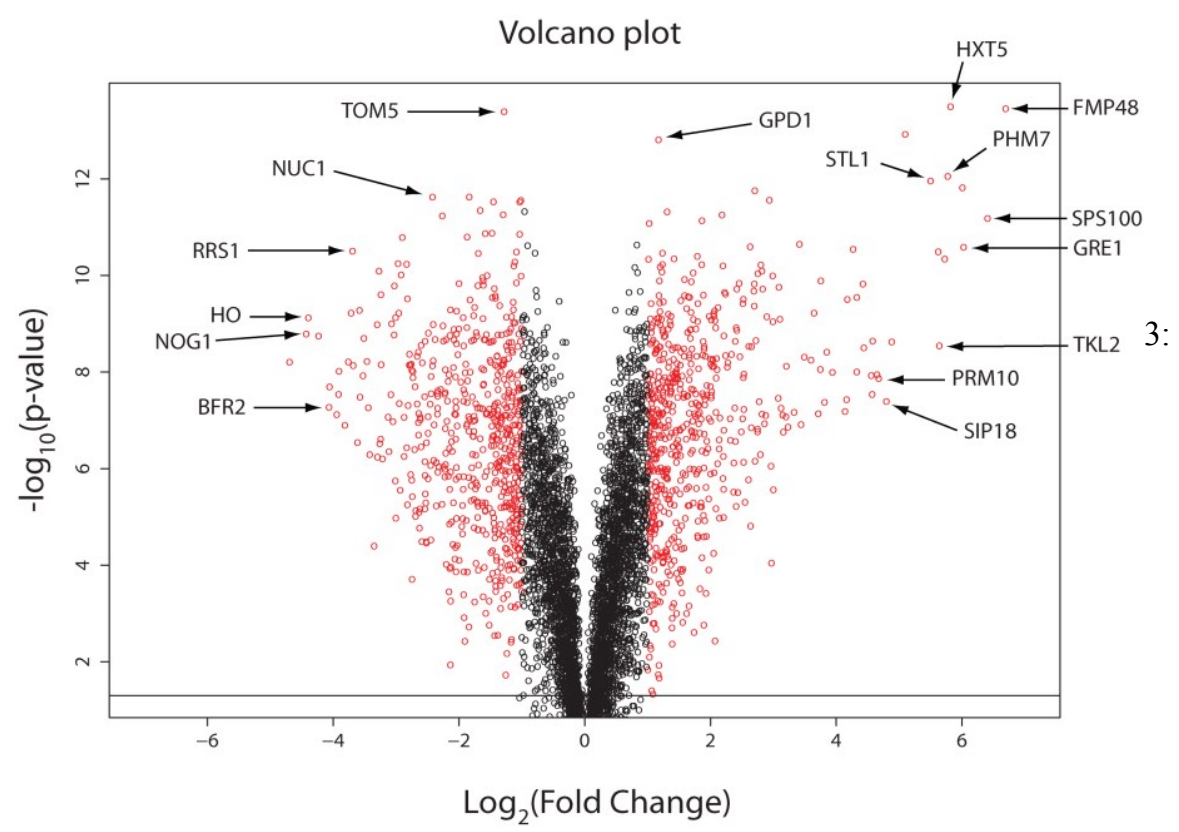




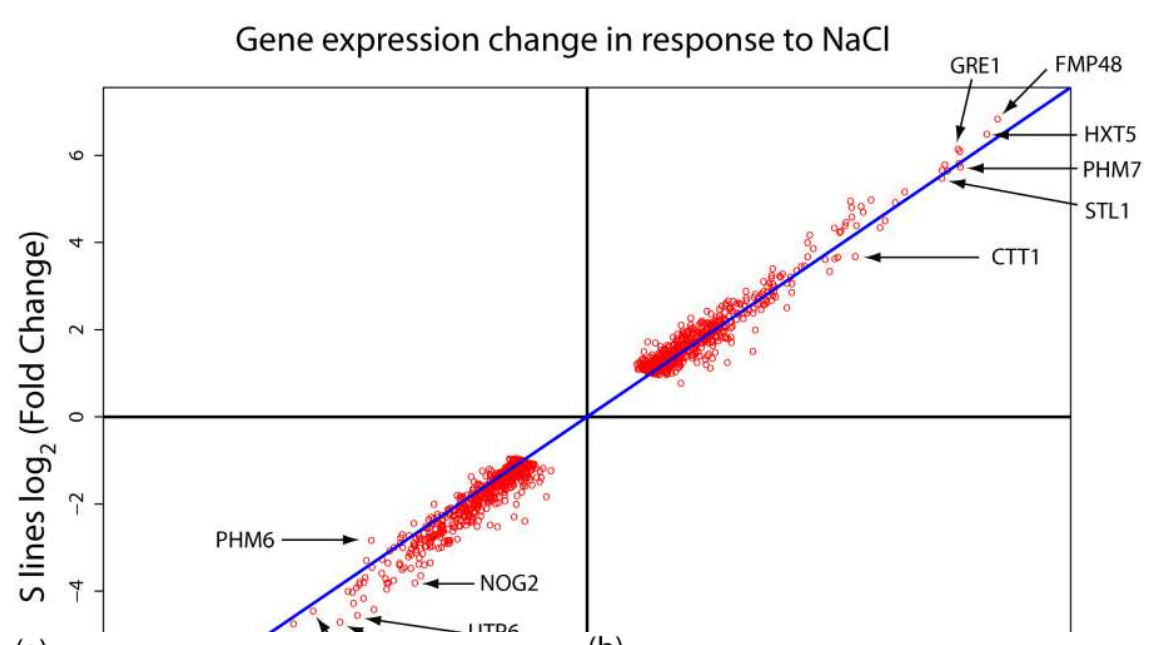

(a)

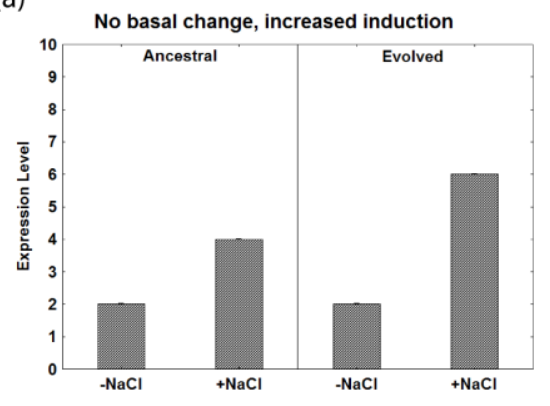

(c)

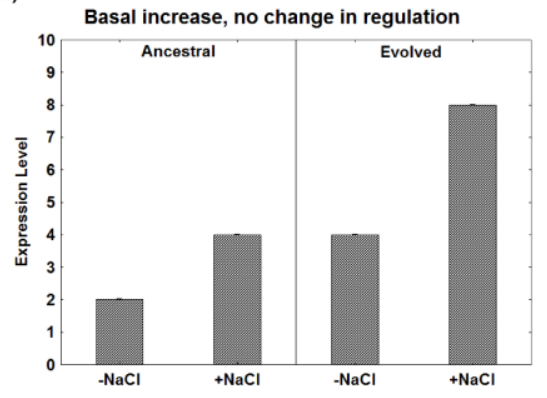

(b)

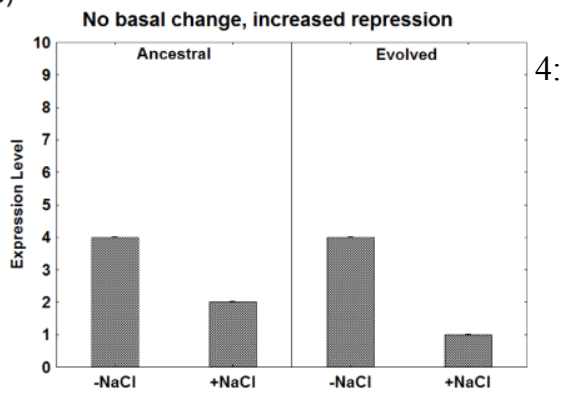

(d)

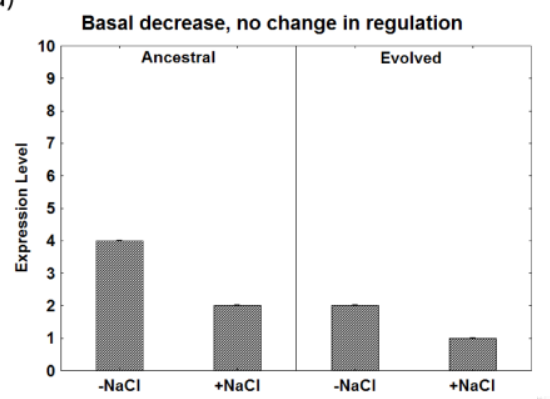


1565 Figure 5:

1566 (a)

1567

1568

1569

1570

1571

1572

1573

1574

1575

1576

1577

1578

1579

1580

1581

1582

1583 (b)

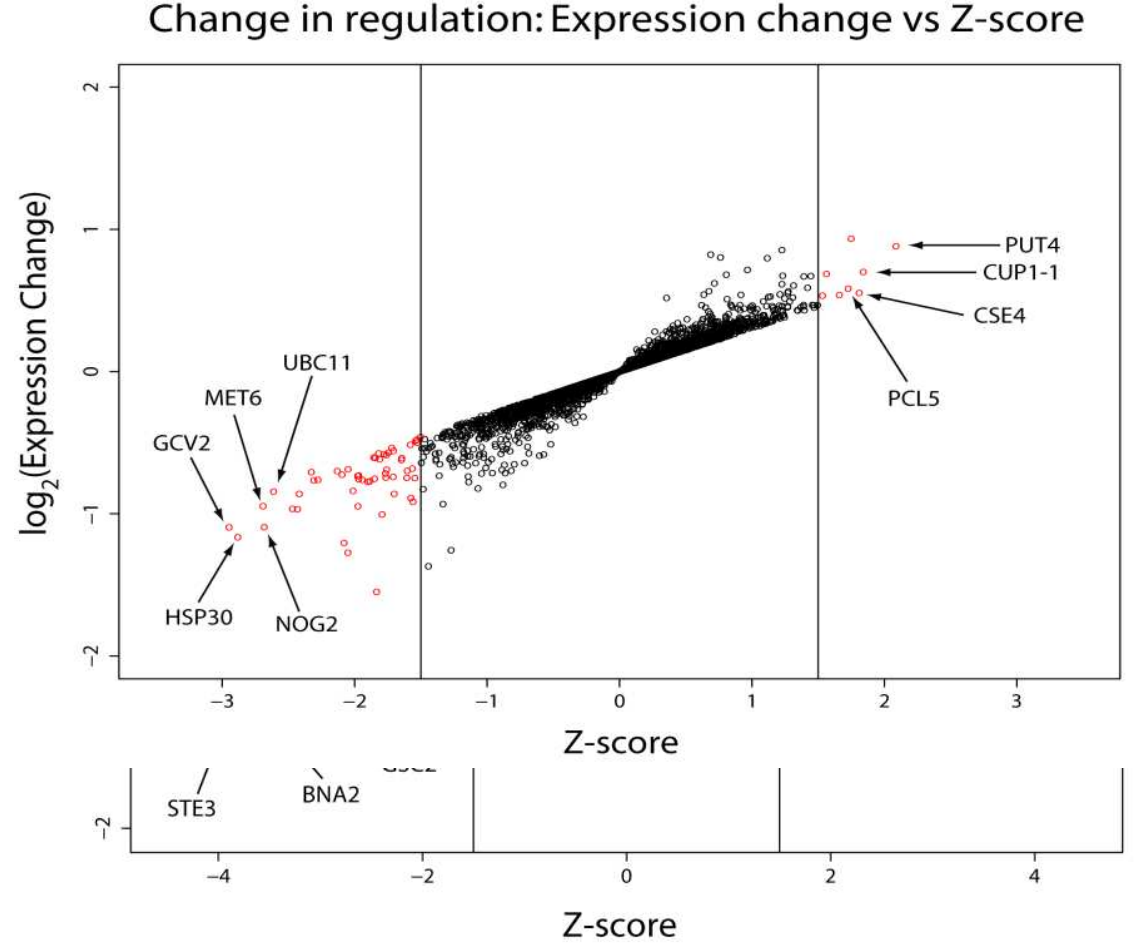


1584

1585

1586

1587

1588

1589

1590

1591

1592

1593

1594

1595

1596

1597

1598

1599

1600

1601

1602 Figure 6: 
(a) Top five genes with change in induction level

\begin{tabular}{|c|c|c|c|c|c|}
\hline Gene & Log2 (Expression Change) & Z-score & Common Name & Type of change & Previous studies \\
\hline YOR348C & 0.8821 & 2.088 & PUT4 & Increased induction & $\begin{array}{c}\text { Posas et al., 2000; Rep et al., 2000; } \\
\text { Yale \& Bonhert, 2001 }\end{array}$ \\
\hline YHR053C & 0.7017 & 1.8401 & CUP1-1 & Increased induction & Yale \& Bonhert, 2001 \\
\hline YKL049C & 0.554 & 1.8101 & CSE4 & New induction & Yale \& Bonhert, 2001 \\
\hline YAR010C & 0.9355 & 1.7493 & -- & New induction & Yale \& Bonhert, 2001 \\
\hline YHR071W & 0.5834 & 1.726 & -- & Increased induction & Yale \& Bonhert, 2001 \\
\hline
\end{tabular}

1612

\section{(b) Top five genes with change in repression level}

1614

1615

1616

1617

1618

1619

1620

1621

1622

1623

1624

1625

1626

1627

1628

1629

\begin{tabular}{|c|c|c|c|c|c|}
\hline Gene & Log2 (Expression Change) & Z-score & Common Name & Type of change & Previous studies \\
\hline YMR189W & -1.0952 & -2.9498 & GCV2 & New repression & Yale \& Bonhert, 2001 \\
\hline YER091C & -0.9458 & -2.6921 & MET6 & Increased repression & Rep et al., 2000 \\
\hline YNR053C & -1.0932 & -2.6838 & NOG2 & Increased repression & -- \\
\hline YOR339C & -0.8424 & -2.613 & UBC11 & Increased repression & -- \\
\hline YHR066W & -0.9644 & -2.4702 & SSF1 & Increased repression & -- \\
\hline
\end{tabular}

(c) Top five genes with increase in basal expression

1630

1631

1632

1633

1634

1635

1636

1637

1638

\begin{tabular}{|c|c|c|c|c|}
\hline Gene & Log2 (Fold Change) & Z-score & Common Name & Previous studies \\
\hline YNL034W & 1.3572 & 4.2657 & - & - \\
\hline YOR384W & 1.2084 & 3.8216 & FRE5 & - \\
\hline YOR049C & 1.4081 & 3.5885 & RSB1 & - \\
\hline YGL192W & 1.5698 & 3.3941 & IME4 & - \\
\hline YOR382W & 1.8809 & 3.3935 & FIT2 & Yale \& Bonhert, 2001 \\
\hline
\end{tabular}

(d) Top five genes with decrease in basal expression

\begin{tabular}{|c|c|c|c|c|}
\hline Gene & Log2 (Fold Change) & Z-score & Common Name & Previous studies \\
\hline YKL178C & -1.2774 & -3.9263 & STE3 & - \\
\hline YML058W-A & -1.2118 & -3.7933 & HUG1 & - \\
\hline YJR078W & -1.2138 & -3.5631 & BNA2 & - \\
\hline YER011W & -1.0741 & -3.361 & TIR1 & - \\
\hline YDR281C & -1.0859 & -2.789 & PHM6 & - \\
\hline
\end{tabular}

1639 Figure 7:

1640 


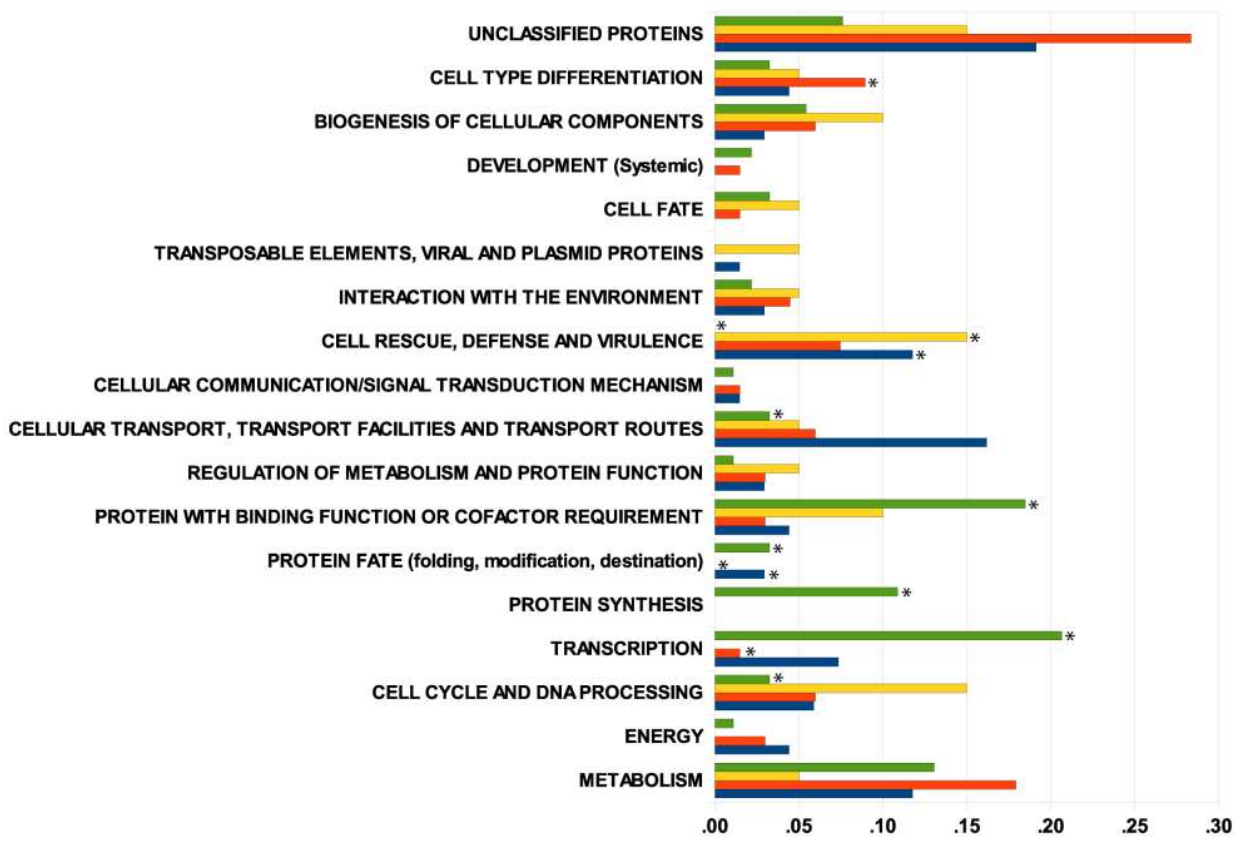

1642

1643

1644

1645

1646

1647

1648

1649

1650

1651

1652

1653

1654

1655

1656

1657

1658

1659

1660

1661 
1668

1669

1670

1671

1672

1673

1674

1675

1676 Figure 8:

(a)

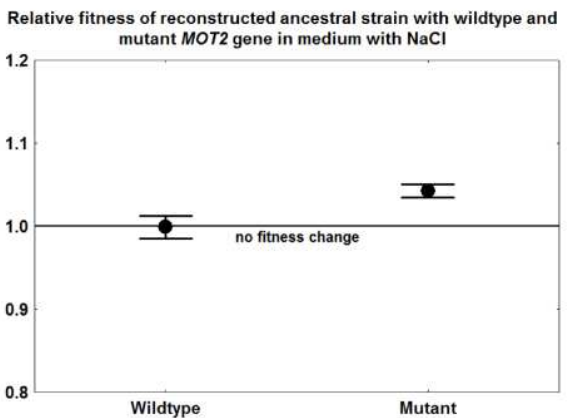

(b)

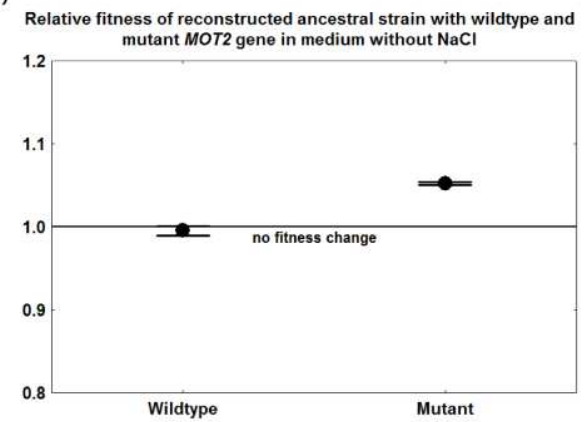

1677

1678

1679

1680

1681

1682

1683

1684

1685

1686

1687

1688

1689

1690

1691

1692

1693 


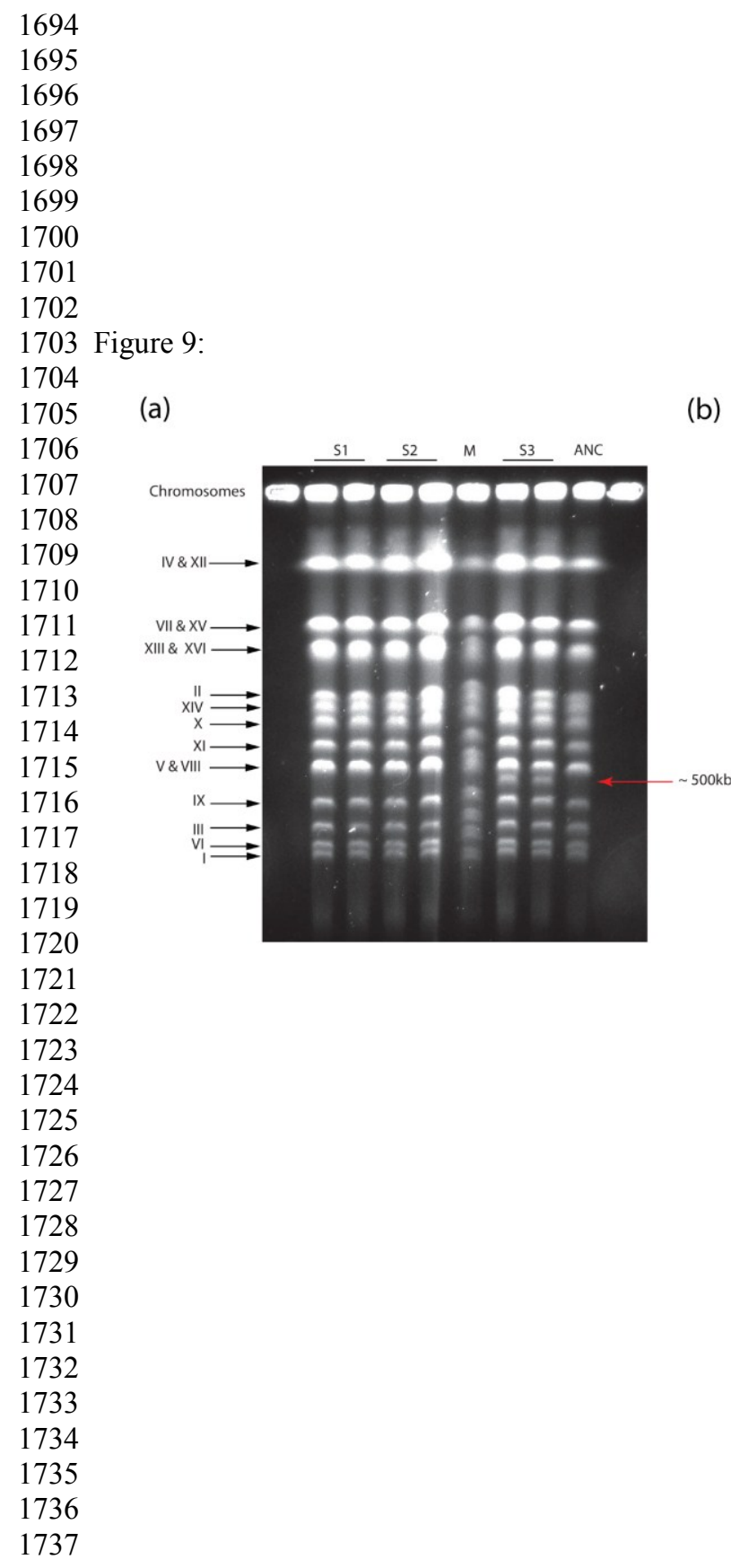

(a)

(b) 
1740 Figure 10:

1741

1742

1743

1744

1745

1746

1747

1748

1749

1750

1751

1752

1753

1754

1755

1756

1757

1758

1759

1760

1761

1762

1763

1764

1765

1766

1767

1768

1769

1770

1771

1772

1773

1774

1775

1776

1777 Figure 11:

1778

1779 (a)

1780

1781
DNA amount per cell of the ancestral strain and the evolved $S$ lines

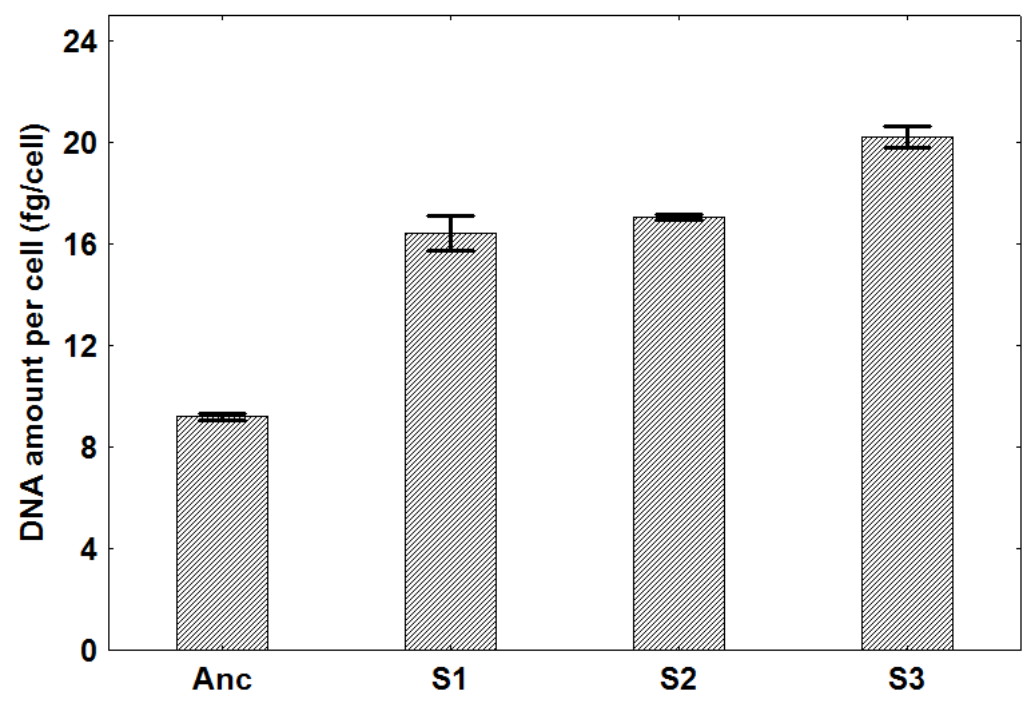

Cell diameter distribution of the ancestral strain and the $\mathbf{S 2}$ line

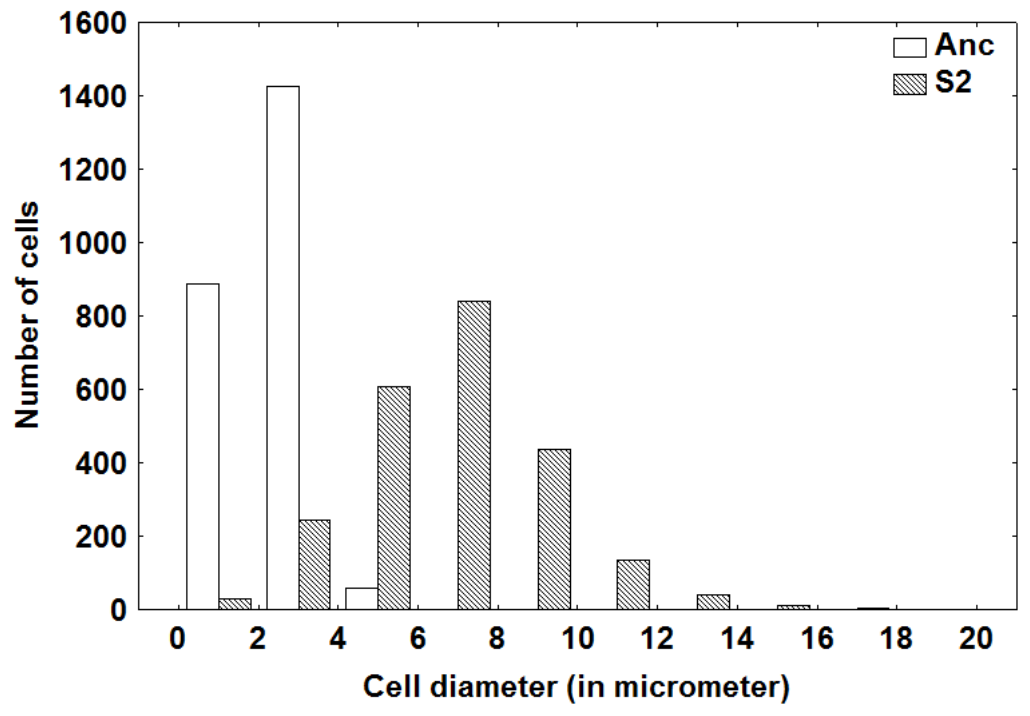


1782

1783

1784

1785

1786

1787

1788

1789

1790

1791

1792

1793

1794

1795

1796 (b)

1797

1798

1799

1800

1801

1802

1803

1804

1805

1806

1807

1808

1809

1810

1811

1812

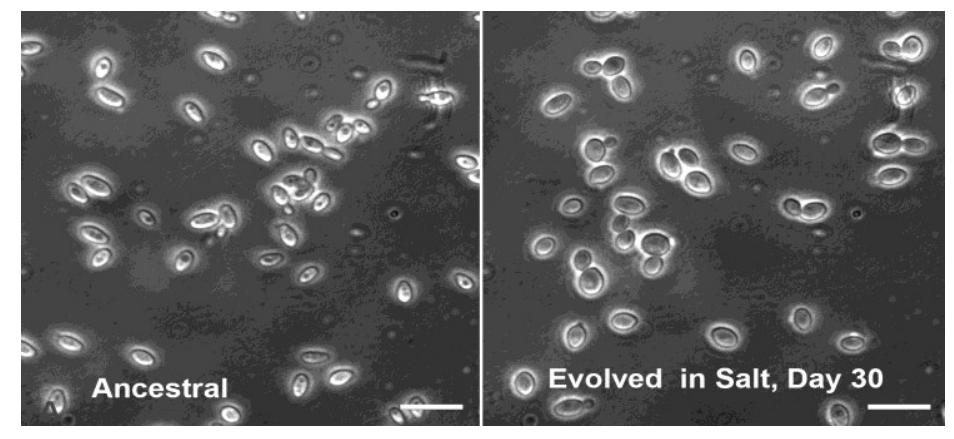

